Pacific Journal of Mathematics

DISTRIBUTIONAL BOUNDARY VALUES IN THE DUAL
SPACES OF SPACES OF TYPE $\mathscr{S}$. 


\title{
DISTRIBUTIONAL BOUNDARY VALUES IN THE DUAL SPACES OF SPACES OF TYPE $\mathscr{S}$
}

\author{
Richard D. Carmichael and E. O. Milton
}

In this paper it is shown that elements of a space of analytic functions defined in the tube domain $T^{C}=\mathbf{R}^{n}+i C$, where $C$ is an open convex cone of a certain type, obtain distributional boundary values in the weak topology of the distribution spaces $\left(\mathscr{S}^{x}\right)^{\prime}, \alpha=\left(\alpha_{1}, \cdots, \alpha_{n}\right), \alpha_{j} \geqq 1, j=1, \cdots, n$; and representation results of the analytic functions in terms of the boundary values are given. Converse results are obtained in which an analytic function in the defined space is constructed from a given distribution in $\left(\mathscr{S}^{x}\right)^{\prime}$, and some applications of the distributional boundary value theorems are obtained. The main results are proved with the aid of several new lemmas concerning the $C^{\infty}$ function spaces of type $\mathscr{S}$ and their dual spaces. The results obtained here are motivated by known results used in the construction of local fields in quantum field theory.

1. Introduction. Gel'fand and Shilov [9] have introduced the spaces of type $\mathscr{S}$, which have been shown to be of importance in at least two areas of applications. Gel'fand and Shilov [10] have used these spaces for a study of the Cauchy problem; while Constantinescu [4] and Rieckers [11] have used them in their studies of quantum field theory.

Constantinescu [4] constructs local fields, which are a category of fields larger than the strictly localizable ones, and proves that the vacuum expectation values in a local field theory are boundary values of functions analytic in a tube domain corresponding to the forward light cone. These vacuum expectation values are in fact distributional boundary values of the analytic functions in the weak topology of the dual spaces of the spaces of type $\mathscr{S}$ and are distributions in these dual spaces.

The present paper is motivated by the distributional boundary value results of Constantinescu [4, Theorems 1 and 2]. We shall generalize these results to functions analytic in tube domains corresponding to open convex cones. The quantum mechanical setting of the tube domain corresponding to the light cone is not the only special case of our generalizations; we shall see that the results corresponding to [4, Theorems 1 and 2] for the important mathematical settings of the half plane in $\mathbf{C}^{1}$ and octant in $\mathbf{C}^{n}$ are also special cases of the theorems we obtain here. We not only obtain generalizations of the known 
results of Constantinescu but also obtain new results concerning distributional boundary values in the dual spaces of the spaces of type $\mathscr{S}$, and we shall obtain new representations of the analytic functions which have these boundary values. One of our main theorems gives more precise information concerning the distributional boundary values obtained by the analytic functions which we consider here than was obtained in a previous paper of one of the authors [2]. Further, we present some mathematical applications of our distributional boundary value results. The main theorems presented in this paper are proved with the aid of several new lemmas which we obtain here concerning the spaces of type $\mathscr{S}$ and their dual spaces.

In $\$ 2$ of this paper we state the notation and definitions needed here. In $\$ 3$ we prove some preliminary lemmas concerning the spaces of type $\mathscr{S}$ and their dual spaces. We define a space of functions whose elements are analytic in tube domains corresponding to open convex cones in $\$ 4$ and prove that these functions obtain distributional boundary values in the topology of the dual spaces of the spaces of type $\mathscr{S}$. Converse results to those of $\S 4$ will be obtained in $\S 5$; while $\S 6$ will contain some applications.

2. Notation and definitions. The $n$-dimensional notation to be used in this paper will be the same as in Carmichael [2], [3]. We recall some of the more important notation for the convenience of the reader. $\langle t, y\rangle=t_{1} y_{1}+\cdots+t_{n} y_{n}, t \in \mathbf{R}^{n}, y \in \mathbf{R}^{n}$, with a similar definition for $\langle t, z\rangle, t \in \mathbf{R}^{n}, z \in \mathbf{C}^{n}$. By $D^{\gamma}, \gamma$ being an $n$-tuple of nonnegative integers, we mean the differential operator $D^{\gamma}=D_{1}^{\gamma_{1}} \cdots D_{n}^{\gamma_{n}}$, where $D_{j}=(1 / 2 \pi i)\left(\partial / \partial t_{j}\right)$ or $D_{j}=(1 / 2 \pi i)\left(\partial / \partial z_{j}\right), j=1, \cdots, n$. We put $D_{t}^{\gamma}$ or $D_{z}^{\gamma}$ to distinguish between differentiating on the real variable $t$ or the complex variable $z$. Let $W=\left(w_{1}, \cdots, w_{n}\right)$ be an $n$-tuple of integers. We define $t^{w}=t_{1}^{w_{1}} \cdots t_{n}^{w_{n}}, t \in \mathbf{R}^{n}$, with a similar definition for $z^{w}, z \in \mathbf{C}^{n}$. We define $|W|=w_{1}+\cdots+w_{n}$ and $W !=w_{1} ! \cdots w_{n} !$.

Throughout this paper $\alpha=\left(\alpha_{1}, \cdots, \alpha_{n}\right)$ will denote an $n$-tuple of positive real numbers. The space of functions $\mathscr{S}_{\alpha}$ consists of the infinitely differentiable functions $\phi$ such that for each $n$-tuple $W=$ $\left(w_{1}, \cdots, w_{n}\right)$ and each $n$-tuple $\gamma$ of nonnegative integers the inequality

$$
\left|t^{W} D^{\gamma} \phi(t)\right| \leqq M_{\gamma}\left(A_{1}^{w_{1}} \cdots A_{n}^{w_{n}}\right)\left(r_{1}^{w_{1} \alpha_{1}} \cdots r_{n}^{w_{n} \alpha_{n}}\right)
$$

is satisfied, where $r_{j}=0,1,2, \cdots, j=1, \cdots, n$, (for $r_{j}=0$, the expression $r_{j}^{w_{j} \alpha_{j}}$ is considered to equal $1, j=1, \cdots, n$. $) \quad A=\left(A_{1}, \cdots, A_{n}\right)$ is a constant $n$-tuple of positive real numbers depending on $\phi$, and $M_{\gamma}$ is a constant real number depending on $\phi$ and $\gamma$. The set of functions $\phi \in \mathscr{S}_{\alpha}$ for which the constants $A_{1}=\bar{A}_{1}, \cdots, A_{n}=\bar{A}_{n}$ may be selected arbitrarily greater than a fixed $A=\left(A_{1}, \cdots, A_{n}\right)$ will be denoted by 
$\mathscr{S}_{\alpha, A}$. We emphasize to the reader that all the properties and definitions which we use here concerning these spaces may be found in Gel'fand and Shilov [9, chapter IV]; and we note that all the results and properties proved in [9, pp. 166-237] for one dimension hold equally well for $n$-dimensions as stated in [9, chapter IV, §9]. We note in particular that $\mathscr{S}_{v}=\cup_{A} \mathscr{I}_{r, A}$.

The space $\mathscr{S}_{r, A}$ may also be defined as the space of $C^{\infty}$ functions such that for each $n$-tuple $\gamma$ of nonnegative integers the inequality

$$
\left|D^{\gamma} \phi(t)\right| \leqq M_{\gamma \delta}^{\prime} \exp \left[-\left(a_{1}-\delta_{1}\right)\left|t_{1}\right|^{\left(\alpha_{1}\right)-1}-\cdots-\left(a_{n}-\delta_{n}\right)\left|t_{n}\right|^{\left(\alpha_{n}\right)-1}\right]
$$

is satisfied for all $\delta=\left(\delta_{1}, \cdots, \delta_{n}\right), \delta_{j}>0, j=1, \cdots, n$, where $M_{\gamma \delta}^{\prime}$ is a constant and

$$
a_{1}=\frac{\alpha_{l}}{e\left(A_{\jmath}\right)^{\left(1 / \alpha_{j}\right)}}, \quad j=1, \cdots, n \text {. }
$$

We put

$$
M_{p}(t)=\exp \left[\left(1-\frac{1}{p}\right)\left(a_{1}\left|t_{1}\right|^{\left(\alpha_{1}\right)-1}+\cdots+a_{n}\left|t_{n}\right|^{\left(\alpha_{n}\right)-1}\right)\right]
$$

$p=2,3,4, \cdots$, where $a_{1}$ and $A_{j}, j=1, \cdots, n$, are related by (1). Then

$$
\|\phi\|_{p}=\left|\underset{t \in \mathbb{R}^{n}}{\sup }\right| \leqq p M_{p}(t)\left|D^{\gamma} \phi(t)\right|, \quad p=2,3,4, \cdots,
$$

defines a sequence of norms on $\mathscr{S}_{x, A}$; and $\mathscr{S}_{x, A}$ may be characterized as the infinitely differentiable functions for which $\|\phi\|_{p}$ is finite for any $p=2,3,4, \cdots$. The space $\mathscr{S}_{r . A}$ belongs to the class of spaces $K\left\{M_{p}\right\}[\boldsymbol{9}$, p. 179] and is a complete countably normed space.

A sequence $\left\{\phi_{r}\right\}$ of elements in $\mathscr{S}_{r . A}$ converges to zero in $\mathscr{S}_{r, A}$ if (i) the norms $\left\|\phi_{v}\right\|_{p}$ are bounded independently of $v$ for any $p=2,3,4, \cdots$ and (ii) for any $n$-tuple $\gamma$ of nonnegative integers, the sequence $\left\{D_{t}^{\gamma} \phi_{v}(t)\right\}$ converges to zero uniformly in any segment $\left\{t:\left|t_{j}\right| \leqq d_{j}<\infty\right.$, $j=1, \cdots, n\}$. A sequence $\left\{\phi_{v}\right\}$ of elements of $\mathscr{S}_{\alpha}$ converges to zero in $\mathscr{S}_{\alpha}$ if (i) there exists an $A$ such that $\phi_{v} \in \mathscr{S}_{\alpha, A}$ for every $v$ and (ii) $\left\{\phi_{v}\right\}$ converges to zero in $\mathscr{S}_{r, A}$ for this value of $A$.

We define the Fourier transform for $L^{1}$ functions $\phi(t)$ by

$$
\hat{\phi}(t)=\mathscr{F}[\phi(t) ; x]=\int_{\mathbf{R}^{n}} \phi(t) e^{2 \pi l(x, t)} d t ;
$$

while the inverse Fourier transform is 


$$
\mathscr{F}^{-1}[\phi(t) ; x]=\int_{\mathbf{R}^{n}} \phi(t) e^{-2 \pi i\langle x, t\rangle} d t .
$$

Let $\alpha$ and $A$ be $n$-tuples as previously stated in this section. The spaces $\mathscr{S}^{x}$ and $\mathscr{S}^{x, A}$ are defined in [9, chapter IV, $\S \S 2.2,3.2$, and 9]. We note that $\mathscr{S}^{x}=\mathscr{F}\left[\mathscr{S}_{x}\right]$ and $\mathscr{S}^{x, A}=\mathscr{F}\left[\mathscr{S}_{x, A}\right]$; that is $\mathscr{S}^{x}$ is the Fourier transform space of $\mathscr{S}_{x}$ and $\mathscr{S}^{x, A}$ is the Fourier transform space of $\mathscr{S}_{x, A}$. We further note that if $\phi(t) \in \mathscr{S}_{x}$, then $\psi(x)=\mathscr{F}[\phi(t) ; x] \in \mathscr{S}^{x}$, and $\phi$ can be recovered from $\psi$ by $\phi(t)=\mathscr{F}^{-1}[\psi(x) ; t]$. The same situation holds for the spaces $\mathscr{S}_{x, A}$ and $\mathscr{S}^{x, A}$. In fact the Fourier transform is a continuous, linear, one-one mapping of $\mathscr{S}_{x}$ onto $\mathscr{S}^{x}$ and $\mathscr{S}_{x, A}$ onto $\mathscr{S}^{x, A}$ with the same being true of the inverse Fourier transform mapping $\mathscr{S}^{x}$ onto $\mathscr{S}_{x}$ and $\mathscr{S}^{x, A}$ onto $\mathscr{S}_{x, A}$. For the notions of convergence in $\mathscr{S}^{x}$ and $\mathscr{S}^{x, A}$ we refer to [9, chapter IV].

The dual spaces of $\mathscr{S}_{r}, \mathscr{S}_{\mathrm{r} . A}, \mathscr{S}^{x}$, and $\mathscr{S}^{x, A}$ are denoted $\mathscr{S}_{\alpha}^{\prime}, \mathscr{S}_{\alpha, A}^{\prime}$, $\left(\mathscr{S}^{x}\right)^{\prime}$, and $\left(\mathscr{S}^{x \cdot A}\right)^{\prime}$, respectively. It is known [4, p. 296] that $\left(\mathscr{S}^{x}\right)^{\prime}=$ $\mathscr{F}\left[\mathscr{S}_{\alpha}^{\prime}\right]$ and $\left(\mathscr{S}^{x, A}\right)^{\prime}=\mathscr{F}_{F}\left[\mathscr{S}_{\alpha, A}^{\prime}\right]$. That is $\left(\mathscr{S}^{x}\right)^{\prime}$ is the Fourier transform space of $\mathscr{S}_{\alpha}^{\prime}$, and $\left(\mathscr{S}^{x, A}\right)^{\prime}$ is the Fourier transform space of $\mathscr{S}_{\alpha, A}^{\prime}$. We recall the definition of this distributional Fourier transform because it will be of extreme importance in this paper. The Fourier transform of an element $V \in \mathscr{S}_{\alpha}^{\prime}$ is defined to be the element $U$ such that the Parseval relation.

$$
\langle U, \psi\rangle=\langle V, \phi\rangle, \quad \phi \in \mathscr{S}_{x}, \quad \psi=\hat{\phi} \in \mathscr{S}^{x},
$$

holds. The element $U$ so defined from $V \in \mathscr{S}_{\alpha}^{\prime}$ is in $\left(\mathscr{S}^{x}\right)^{\prime}$ and is the Fourier transform, denoted $U=\mathscr{F}[V]$, of $V$. (4) is a valid definition because of the relation between $\mathscr{S}_{v}$ and $\mathscr{S}^{x}$ via the Fourier transform; and in fact the Fourier transform defined by (4) is a continuous, linear, one-to-one mapping of $\mathscr{S}_{\alpha}^{\prime}$ onto $\left(\mathscr{S}^{x}\right)$.

The definition of the spaces of $C^{x}$ functions $\mathscr{E}, \mathscr{D}$, and $\mathscr{S}$ and the spaces of distributions $\mathscr{D}^{\prime}$ and $\mathscr{S}^{\prime}$ can be found in Schwartz [12]. We have the containments $\mathscr{D} \subset \mathscr{S}_{x} \subset \mathscr{S}, \mathscr{S}^{\prime} \subset \mathscr{S}_{\alpha}^{\prime} \subset \mathscr{D}^{\prime}$, and $\mathscr{S}^{\prime} \subset \mathscr{S}_{a, A}^{\prime} \subset \mathscr{D}^{\prime}$ [4, p. 296]. $\operatorname{supp}(f)$ and $\operatorname{supp}(V)$ denote the supports of the function $f$ and the distribution $V$, respectively. (See Schwartz [12] for the support of a distribution.)

The set $C \subset \mathbf{R}^{n}$ is a cone (with vertex at zero) if $y \in C$ implies $\mu y \in C$ for all positive scalars $\mu$. The intersection of the cone $C$ with the unit sphere is called the projection of $C$ and is denoted $\operatorname{pr}(C)$. Let $C^{\prime}$ be an open cone such that $\operatorname{pr}\left(\bar{C}^{\prime}\right) \subset \operatorname{pr}(C)$; then $C^{\prime}$ will be called a compact subcone of $C$. The function

$$
u_{C}(t)=\sup _{y \in \operatorname{pr}(C)}(-\langle t, y\rangle), t \in \mathbf{R}^{n},
$$


is the indicatrix of the cone $C$, and the set $C^{*}=\left\{t: u_{C}(t) \leqq 0\right\}=$ $\{t:\langle y, t\rangle \geqq 0, y \in C\}$ is the dual cone of the cone $C$. The number

$$
\rho_{C}=\sup _{t \in C^{*}} \frac{u_{O(C)}(t)}{u_{C}(t)}, C_{*}=\mathbf{R}^{n} \backslash C^{*},
$$

characterizes the nonconvexity of the cone $C$, where $O(C)$ denotes the convex envelope of $C$. Throughout this paper $S_{b}$ will denote the set $S_{b}=\left\{t: u_{C}(t) \leqq b\right\}$ where $b \geqq 0$; and $T^{C}$ will be the set $\mathbf{R}^{n}+i C \subset \mathbf{C}^{n}$. If the cone $C$ is open and connected, $T^{C}$ will be called a tubular radial domain.

In this paper we shall use some terminology from the theory of topological vector spaces and their dual spaces such as bounded set in a topological vector space, bounded set in a vector space which is the union of topological vector spaces, strongly bounded set in a dual space, and strongly continuous mapping between two dual spaces. We refer to Edwards [6] and Friedman [7, Chapter 1] for this terminology.

Let $C$ be an open connected cone, and let $C^{\prime}$ be an arbitrary compact subcone of $C$. Let $f(z)$ be a function of $z=x+i y \in T^{c}$; and let $U$ be a generalized function. By $f(z) \rightarrow U$ in the weak topology of the generalized function space as $y=\operatorname{Im}(z) \rightarrow 0$ (i.e., $y_{j} \rightarrow 0$, $j=1, \cdots, n), y \in C^{\prime} \subset C$, we mean $\langle f(z), \psi(x)\rangle \rightarrow\langle U, \psi\rangle$ as $y \rightarrow 0, y \in$ $C^{\prime} \subset C$, for each fixed element $\psi$ in the corresponding test function space. We then call $U$ the (weak) distributional boundary value of $f(z)$; and we note that it is defined on the distinguished boundary of $T^{c}$, $\left\{z=x+i y: x \in \mathbf{R}^{n}, y=(0, \cdots, 0)\right\}$, which is not necessarily the topological boundary of $T^{C}$.

We are concerned in this paper with analytic functions defined in tubular radial domains $T^{C}$ or $T^{C^{\prime}}$, where $C$ is an open connected cone and $C^{\prime}$ is an arbitrary compact subcone of $C$. Bochner's analytic extension theorem [1, Chapter 5] states that any function which is analytic in such a tube domain has an analytic extension to the convex envelope of the tube domain. In light of this result it suffices to assume for our purposes in this paper that the open connected cone $C$ is in fact convex and that the compact subcones of $C$ are convex. This assumption will be made in the remainder of this paper.

3. Preliminary lemmas. In this section we shall obtain some lemmas concerning the spaces of type $\mathscr{S}$ and their dual spaces which will be useful in the succeeding sections. We begin by proving a series of lemmas concerning the spaces of type $\mathscr{S}, \mathscr{S}_{x}$.

Lemma 1. Let $\Phi \subseteq \mathscr{E}$ be a set of functions such that for each n-tuple $\gamma$ of nonnegative integers $\left\{D^{\gamma} \phi(t): \phi \in \Phi\right\}$ is uniformly 
bounded. Let $\Psi$ be a bounded set in $\mathscr{S}_{\alpha}$. Then $\Phi \Psi=\{\phi \psi: \phi \in \Phi$, $\psi \in \Psi\}$ is a bounded set in $\mathscr{S}_{\alpha}$.

Proof. The space $\mathscr{S}_{\alpha}$ can be constructed as $\cup_{A} \mathscr{S}_{\alpha, A}[9, \mathrm{p} .178, \mathrm{p}$. 238], where $A=(n, \cdots, n), n=1,2,3, \cdots$. The spaces $\mathscr{S}_{\alpha, A}$ are complete countably normed spaces, and $\mathscr{S}_{x, A} \subset \mathscr{S}_{x, B}$ if $A_{j}<B_{j}, j=$ $1, \cdots, n$. Thus there exists an $n$-tuple $\bar{A}=\left(\bar{A}_{1}, \cdots, \bar{A}_{n}\right)$ such that $\Psi$ is a bounded set in $\mathscr{S}_{r, \bar{A}}$ since $\Psi$ is a bounded set in $\mathscr{S}_{\mathrm{r}}$. It is obvious that $\Phi \Psi \subset \mathscr{E}$. Let $p=2,3,4, \cdots$ be arbitrary but fixed. For the fixed $p$, let $\gamma$ be an arbitrary $n$-tuple of nonnegative integers such that $|\gamma| \leqq$ $p$. Let $\phi \in \Phi$ and $\psi \in \Psi$. Choose the $n$-tuple $\left(a_{1}, \cdots, a_{n}\right)$ from the $n$-tuple $\bar{A}$ according to (1) and recall the function $M_{p}(t)$ from (2). Using the generalized Leibnitz rule we have

$$
M_{p}(t) D_{t}^{\gamma}(\phi(t) \psi(t))=M_{p}(t) \sum_{\beta+\rho=\gamma} \frac{\gamma !}{\beta ! \rho !} D^{\beta} \phi(t) D^{\rho} \psi(t),
$$

where $\beta$ and $\rho$ are $n$-tuples of nonnegative integers. Letting $N_{\beta}$ denote the uniform bound on $\left\{D^{\beta} \phi(t): \phi \in \Phi\right\}$ and recalling that $\gamma$ is an arbitrary $n$-tuple of nonnegative integers such that $|\gamma| \leqq p$, we obtain

$$
\begin{aligned}
M_{p}(t)\left|D_{t}^{\gamma}(\phi(t) \psi(t))\right| & \leqq M_{p}(t) \sum_{\beta+\rho=\gamma} \frac{\gamma !}{\beta ! \rho !}\left|D^{\beta} \phi(t)\right|\left|D^{\rho} \psi(t)\right| \\
& \leqq \sum_{\beta+\rho=\gamma} \frac{\gamma !}{\beta ! \rho !} N_{\beta}\left(M_{p}(t)\left|D^{\rho} \psi(t)\right|\right) \\
& \leqq\|\psi\|_{\rho} \sum_{\beta+\rho=\gamma} \frac{\gamma !}{\beta ! \rho !} N_{\beta} .
\end{aligned}
$$

Inequality (5) holds for each $|\gamma|,|\gamma| \leqq p$, and all $t \in \mathbf{R}^{n}$. Thus

$$
\|\phi \psi\|_{p} \leqq\|\psi\|_{p} \sup _{|\gamma| \leqq p}\left(\sum_{\beta+\rho=\gamma} \frac{\gamma !}{\beta ! \rho !} N_{\beta}\right)
$$

Since $p=2,3,4, \cdots$ is arbitrary and $\psi \in \Psi \subset \mathscr{S}_{\alpha, \bar{A}}$, then (6) shows that $\Phi \Psi \subset \mathscr{S}_{\alpha, \bar{A} .}$. Further (6) proves that $\Phi \Psi$ is a bounded set in $\mathscr{S}_{\alpha, \bar{A}}$ since $\Psi$ is a bounded set in $\mathscr{S}_{\alpha, \bar{A} \text {. }}$. Thus $\Phi \Psi$ is a bounded set in $\mathscr{S}_{\alpha}$, and the proof is complete.

As we have noted in $\S 2$, the $\mathscr{S}_{\alpha, A}$ spaces belong to the class of $K\left\{M_{p}\right\}$ spaces of Gel'fand and Shilov [9, p. 177], where $M_{p}(t)$ is defined in (2); and Gel'fand and Shilov [9, p. 177] prove that condition (P) of [9, p. 92] is satisfied. Further, it is easily seen that both conditions (M) and (N) of [9, p. 111] are also satisfied. Thus by [9, pp. 111-112], the system of norms in (3) is equivalent to the system of norms 


$$
\|\phi\|_{p}^{\prime}=\sup _{|\gamma| \leqq p} \int_{\mathbf{R}^{n}} M_{p}(t)\left|D^{\gamma} \phi(t)\right| d t, \quad p=2,3,4, \cdots
$$

in $\mathscr{S}_{x, A}$, where $M_{p}(t)$ is the function defined in (2). Using this fact we now prove the following lemma.

Lemma 2. Let $C$ be an open convex cone. Let $\left\{\psi_{y}(t): y \in C\right\} \subset \mathscr{E}$ such that for each n-tuple $\gamma$ of nonnegative integers $\left\{D_{t}^{\gamma}\left(\psi_{y}(t)\right): y \in C\right\}$ is uniformly bounded and $D_{t}^{\gamma}\left(\psi_{y}(t)\right) \rightarrow 0$ pointwise for $t \in \mathbf{R}^{n}$ as $y \rightarrow 0$, $y \in C$. Then for each $\phi \in \mathscr{S}_{\alpha}, \phi \psi_{y} \rightarrow 0$ in $\mathscr{S}_{\alpha}$ as $y \rightarrow 0, y \in C$.

Proof. Let $\phi$ be an arbitrary but fixed element in $\mathscr{S}_{\alpha}$. As in the proof of Lemma 1, there exists an $n$-tuple $A$ such that $\phi \in \mathscr{S}_{\alpha, A}$. Let $p=2,3,4, \cdots$ be arbitrary but fixed. For the fixed $p$, let $\gamma$ be an arbitrary $n$-tuple of nonnegative integers such that $|\gamma| \leqq p$. It is obvious that $\phi \psi_{y} \in C^{\infty}$ for each $y \in C$; and using the generalized Leibnitz rule we have

(8) $M_{p}(t)\left|D_{t}^{\gamma}\left(\phi(t) \psi_{y}(t)\right)\right| \leqq M_{p}(t) \sum_{\beta+\rho=\gamma} \frac{\gamma !}{\beta ! \rho !}\left|D^{\beta} \phi(t)\right|\left|D_{t}^{\rho}\left(\psi_{y}(t)\right)\right|$,

where $M_{p}(t)$ is the function in (2) and $\beta$ and $\rho$ are $n$-tuples of nonnegative integers. Now let $N_{\rho}$ denote the uniform bound on $\left\{D_{t}^{\rho}\left(\psi_{y}(t)\right): y \in C\right\}$, and recall that $\gamma$ is an arbitrary $n$-tuple of nonnegative integers such that $|\gamma| \leqq p$. From (8) we obtain

(9)

$$
M_{p}(t)\left|D_{t}^{\gamma}(\phi(t))\right| \leqq \sum_{\beta+\rho=\gamma} \frac{\gamma !}{\beta ! \rho !} N_{p}\left(M_{p}(t)\left|D^{\gamma} \phi(t)\right|\right)
$$

$$
\leqq\|\phi\|_{p} \sum_{\beta+\rho=\gamma} \frac{\gamma !}{\beta ! \rho !} N_{\rho}
$$

This inequality holds for all $\gamma,|\gamma| \leqq p$, and all $t \in \mathbf{R}^{n}$. It thus follows from (9) that

$$
\left\|\phi \psi_{y}\right\|_{p} \leqq\|\phi\|_{p} \sup _{|\gamma| \leqq p}\left(\sum_{\beta+\rho=\gamma} \frac{\gamma !}{\beta ! \rho !} N_{\rho}\right)
$$

and this bound is independent of $y \in C$. Since $p=2,3,4, \cdots$ is arbitrary and $\phi \in \mathscr{S}_{\alpha, A},(10)$ shows that $\left(\phi \psi_{y}\right) \in \mathscr{S}_{\alpha, A}$ for each $y \in C$ and the norms $\left\|\phi \psi_{y}\right\|_{p}, p=2,3,4, \cdots$, are uniformly bounded independent of $y \in C$. 
It remains to prove that $\left\{D_{t}^{\gamma}\left(\phi(t) \psi_{y}(t)\right)\right\}$ converges to zero uniformly in any segment $\left\{t:\left|t_{j}\right| \leqq d_{j}<\infty, j=1, \cdots, n\right\}$ for each $n$-tuple $\gamma$ of nonnegative integers as $y \rightarrow 0, y \in C$. To do this it suffices to show that

$$
\lim _{\substack{y \rightarrow 0 \\ y \in C}}\left\|\phi \psi_{y}\right\|_{p}=0, \quad \quad p=2,3,4, \cdots
$$

from which it is easily seen that we can actually conclude $\left\{D_{t}^{\gamma}\left(\phi(t) \psi_{y}(t)\right)\right\}$ converges to zero uniformly on the whole of $\mathbf{R}^{n}$ for each $n$-tuple $\gamma$ as $y \rightarrow 0, y \in C$. To prove (11) it suffices to show

$$
\lim _{\substack{y \rightarrow 0 \\ y \in C}}\left\|\phi \psi_{y}\right\|_{p}^{\prime}=0, \quad \quad p=2,3,4, \cdots
$$

since the system of norms defined in (3) and (7) are equivalent in $\mathscr{S}_{\alpha, A}$. Thus we again let $p=2,3,4, \cdots$ be arbitrary but fixed and $\gamma$ be an $n$-tuple of nonnegative integers such that $|\gamma| \leqq p$. For the fixed $p$ we put

$$
q=\frac{1}{p(p+1)}
$$

Then

$$
M_{p}(t)=M_{p+1}(t) \exp \left(-q\left(a_{1}\left|t_{1}\right|^{\left.\mid \alpha_{1}\right)^{-1}}+\cdots+a_{n}\left|t_{n}\right|^{\left(\alpha_{n}\right)^{-1}}\right)\right) .
$$

Thus

$$
\begin{gathered}
M_{p}(t)\left|D_{t}^{\gamma}\left(\phi(t) \psi_{y}(t)\right)\right| \\
\leqq M_{p+1}(t) \exp \left(-q\left(a_{1}\left|t_{1}\right|^{\left(\alpha_{1}\right)^{-1}}+\cdots+a_{n}\left|t_{n}\right|^{\mid\left(\alpha_{n}\right)^{-1}}\right)\right) \\
\cdot \sum_{\beta+\rho=\gamma} \frac{\gamma !}{\beta ! \rho !}\left|D^{\beta} \phi(t)\right|\left|D_{t}^{\rho}\left(\psi_{y}(t)\right)\right| .
\end{gathered}
$$

Again let $N_{\rho}$ denote the uniform bound on $\left\{D_{t}^{\rho}\left(\psi_{y}(t)\right): y \in C\right\}$ and recall that $\gamma$ is arbitrary such that $|\gamma| \leqq p$. From (13) we obtain

$$
M_{p}(t)\left|D_{t}^{\gamma}\left(\phi(t) \psi_{y}(t)\right)\right|
$$

$$
\leqq\|\phi\|_{p+1} \exp \left(-q\left(\left|t_{1}\right|^{\left(\alpha_{1}\right)^{-1}}+\cdots+a_{n}\left|t_{n}\right|^{\left(\alpha_{n}\right)^{-1}}\right)\right)\left(\sum_{\beta+\rho=\gamma} \frac{\gamma !}{\beta ! \rho !} N_{\rho}\right) .
$$


Since $\phi \in \mathscr{S}_{\alpha, A}$ implies $\|\phi\|_{\rho+1}<\infty$, we see that the right hand side of (14) is an element of $L^{\prime}$ and bounds the left hand side independently of $y \in C$. Further from (8) we have

$$
M_{p}(t)\left|D_{t}^{\gamma}\left(\phi(t) \psi_{y}(t)\right)\right| \leqq H \phi \|_{p} \sum_{\beta+\rho=\gamma} \frac{\gamma !}{\beta ! \rho !}\left|D_{t}^{\rho}\left(\psi_{y}(t)\right)\right|
$$

The hypothesis that $D_{t}^{\gamma}\left(\psi_{y}(t)\right) \rightarrow 0$ pointwise on $\mathbf{R}^{n}$ as $y \rightarrow 0, y \rightarrow C$, for each $\gamma$ and (15) show that $\left(M_{p}(t)\left|D_{t}^{\gamma}\left(\phi(t) \psi_{y}(t)\right)\right|\right) \rightarrow 0$ pointwise on $\mathbf{R}^{n}$ as $y \rightarrow 0, y \in C$. An application of the Lebesgue dominated convergence theorem now yields

$$
\lim _{\substack{y \rightarrow 0 \\ y \in C}} \int_{\mathbf{R}^{n}} M_{p}(t)\left|D_{t}^{\gamma}\left(\phi(t) \psi_{y}(t)\right)\right| d t=0
$$

and (16) holds for each $\gamma,|\gamma| \leqq p$. It thus follows from (16) that $\left\|\phi \psi_{y}\right\|_{p}^{\prime} \rightarrow 0$ as $y \rightarrow 0, y \in C$; and we recall that $p=2,3,4, \cdots$ is arbitrary. Thus (12) holds. By definition of convergence in $\mathscr{S}_{\alpha}$, we have $\phi \psi_{y} \rightarrow 0$ in $\mathscr{S}_{\alpha}$ as $y \rightarrow 0, y \in C$; and the proof is complete.

We note the following restricted version of Lemma 2 .

Lemma 3. Let $C$ be an open convex cone, and let $Q$ be an arbitrary but fixed positive real number. Let $\left\{\psi_{y}(t): y \in C\right\} \subset \mathscr{E}$ such that for each n-tuple $\gamma$ of nonnegative integers $\left\{D_{t}^{\gamma}\left(\psi_{y}(t)\right): y \in C,|y| \leqq Q\right\}$ is uniformly bounded and $D_{t}^{\gamma}\left(\psi_{y}(t)\right) \rightarrow 0$ pointwise for $t \in \mathbf{R}^{n}$ as $y \rightarrow 0$, $y \in C$. Then for each $\phi \in \mathscr{S}_{\alpha}, \phi \psi_{y} \rightarrow 0$ in $\mathscr{S}_{\alpha}$ as $y \rightarrow 0, y \in C$.

It is clear that Lemma 3 is just a special case of Lemma 2 since we are obtaining a convergence result as $y \rightarrow 0, y \in C$. We state Lemma 3 separately because it is the version of this result which we use later in this paper.

Let $b \geqq 0$ be fixed. Let $\xi(\eta) \in \mathscr{E}, \eta \in \mathbf{R}^{1}$, such that $\xi(\eta)=1$, $\eta \geqq(-b), \xi(\eta)=0, \eta \leqq(-b-\epsilon), \epsilon>0$ and fixed, and $0 \leqq \xi(\eta) \leqq$ 1. Put

$$
\lambda(t)=\xi(\langle y, t\rangle), y \in C, t \in \mathbf{R}^{n}
$$

where $C$ is an open convex cone. We have $\lambda(t) \in \mathscr{E}, t \in \mathbf{R}^{n}$.

Lemma 4. Let $C$ be an open convex cone, and let $\phi \in \mathscr{S}_{\alpha}$. Let $\lambda(t)$ be the function defined in (17) where $b \geqq 0$ is arbitrary but fixed. Then $(\lambda(t) \exp (-2 \pi\langle y, t\rangle) \phi(t)) \rightarrow(\lambda(t) \phi(t))$ in $\mathscr{S}_{\alpha}$ as $y \rightarrow 0$, $y \in C$. 
Proof. It suffices to show $(\lambda(t)(\exp (-2 \pi\langle y, t\rangle)-1) \phi(t)) \rightarrow 0$ in $\mathscr{S}_{\alpha}$ as $y \rightarrow 0, y \in C$. To do this we use Lemma 3 with

$$
\psi_{y}(t)=\left(\lambda(t)\left(e^{-2 \pi(y, t)}-1\right)\right), y \in C .
$$

Note first that $\left\{\psi_{y}(t): y \in C\right\} \subset \mathscr{E}$. Let $\gamma$ be an arbitrary $n$-tuple of nonnegative integers, and for the present let $y$ be an arbitrary but fixed point of $C$. Using the generalized Leibnitz rule (recall $D_{j}=(1 / 2 \pi i)$ $\left.\left(\partial / \partial t_{j}\right)\right)$

$$
D_{t}^{\gamma}\left(\psi_{y}(t)\right)=\sum_{\beta+\rho=\gamma} \frac{\gamma !}{\beta ! \rho !} D^{\beta} \lambda(t)\left((i)^{|\rho|} y^{\rho} e^{-2 \pi\langle y, t\rangle}-D^{\rho}(1)\right) .
$$

Now $\exp (-2 \pi\langle y, t\rangle) \rightarrow 1$ pointwise for $t \in \mathbf{R}^{n}$ as $y \rightarrow 0, y \in C$. Since $D^{\beta} \lambda(t)$ is bounded on $\mathbf{R}^{n}$ for each $\beta, \beta+\rho=\gamma$, we have that the term in the sum in (18) which corresponds to $\beta=\gamma$ and $\rho=(0, \cdots, 0)$ satisfies $\left(D^{\gamma} \lambda(t)(\exp (-2 \pi\langle y, t\rangle)-1)\right) \rightarrow 0$ pointwise for $t \in \mathbf{R}^{n}$ as $y \rightarrow 0, y \in$ $C$. In order to obtain a similar convergence in the other terms in the sum in (18), we must recall that by the definition of $\lambda(t), D^{\beta} \lambda(t)=0$ for all $\beta, \beta+\rho=\gamma$, for $t \in \mathbf{R}^{n}$ such that $\langle y, t\rangle \leqq(-b-\epsilon), y \in C$. Thus $D_{t}^{\gamma}\left(\psi_{y}(t)\right)=0$ if $\langle y, t\rangle \leqq(-b-\epsilon), y \in C$; so that it suffices to consider $D_{t}^{\gamma}\left(\psi_{y}(t)\right)$ for $t \in \mathbf{R}^{n}$ such that $\langle y, t\rangle>(-b-\epsilon), y \in C$. For the other terms in the sum in (18), those corresponding to $\rho \neq(0, \cdots, 0), D^{\rho}(1)=0$; and for $\langle y, t\rangle>(-b-\epsilon), t \in C$,

$$
\left|D^{\beta} \lambda(t)(i)^{|\rho|} y^{\rho} e^{-2 \pi(y, t)}\right| \leqq K_{\beta} e^{2 \pi(b+\epsilon)}\left|y^{\rho}\right|
$$

where $b \geqq 0$ and $\epsilon>0$ are fixed and $K_{\beta}$ bounds $\left|D^{\beta} \lambda(t)\right|, t \in \mathbf{R}^{n}$. This estimate combined with the fact that $D_{t}^{\gamma}\left(\psi_{y}(t)\right)=0$ for all $t \in \mathbf{R}^{n}$ such that $\langle y, t\rangle \leqq(-b-\epsilon), y \in C$, shows that all the terms in the sum in (18) corresponding to $\rho \neq(0, \cdots, 0)$ converge to zero pointwise for $t \in \mathbf{R}^{n}$ (in fact uniformly in $t \in \mathbf{R}^{n}$ ) as $y \rightarrow 0, y \in C$. Thus we have that every term in the sum in (18) converges to zero pointwise for $t \in \mathbf{R}^{n}$ as $y \rightarrow 0$, $y \in C$; and from (18) this same convergence holds for $D_{t}^{\gamma}\left(\psi_{y}(t)\right)$ for any $\gamma$.

It remains to show that the set $\left\{D_{f}^{\gamma}\left(\psi_{y}(t)\right): y \in C,|y| \leqq Q\right\}$, where $Q$ is a fixed positive real number, is uniformly bounded for each $n$-tuple $\gamma$ of nonnegative integers. Again recalling that $D_{i}^{\gamma}\left(\psi_{y}(t)\right)=0$ for $t \in \mathbf{R}^{n}$ such that $\langle y, t\rangle \leqq(-b-\epsilon), y \in C$, we obtain from (18)

$$
\left|D_{t}^{\gamma}\left(\psi_{y}(t)\right)\right| \leqq \sum_{\beta+\rho=\gamma} \frac{\gamma !}{\beta ! \rho !} K_{\beta}\left(Q^{|\rho|} e^{2 \pi(b+\epsilon)}+D^{\rho}(1)\right)
$$

$t \in \mathbf{R}^{n}$, where $y \in C$ such that $|y| \leqq Q, K_{\beta}$ bounds $\left|D^{\beta} \lambda(t)\right|$, and $b \geqq 0$ 
and $\epsilon>0$ are fixed. Thus for each $\gamma,\left\{D_{t}^{\gamma}\left(\psi_{y}(t)\right): y \in C,|y| \leqq Q\right\}$ is uniformly bounded (i.e., bounded independently of $t \in \mathbf{R}^{n}$ and $y \in C$ such that $|y| \leqq Q$.) We conclude from that

$$
(\lambda(t) \exp (-2 \pi\langle y, t\rangle)-1) \phi(t)) \rightarrow 0
$$

in $\mathscr{S}_{\alpha}$ as $y \rightarrow 0, y \in C$, which proves the desired result.

LEMMA 5. Let $C$ be an open convex cone, and let $\Psi$ be a bounded set in $\mathscr{S}_{\alpha}$. Let $\lambda(t)$ be the function defined in (17) where $b \geqq 0$ is arbitrary but fixed. Let $\Phi=\{\lambda(t) \exp (-2 \pi\langle y, t\rangle): y \in C,|y| \leqq Q\}$, where $Q$ is an arbitrary but fixed positive real number. Then $\Phi \Psi$ is a bounded set in $\mathscr{S}_{\alpha}$.

Proof. Obviously $\Phi \subset \mathscr{E}$; and from the proof of Lemma 4, $\left\{D_{t}^{\gamma}(\lambda(t) \exp (-2 \pi\langle y, t\rangle)): y \in C,|y| \leqq Q\right\}$ is uniformly bounded for each $n$-tuple $\gamma$ of nonnegative integers. By Lemma $1, \Phi \Psi$ is a bounded set in $\mathscr{S}_{\alpha}$.

In this paper we shall at times consider open convex cones $C$ having the following property which we denote as property $(\mathrm{C})$ :

(C)An open convex cone $C$ has property (C) if for each $\phi \in \mathscr{S}_{\alpha}$ there exists an $n$-tuple $A=\left(A_{1}, \cdots, A_{n}\right), A_{j}>0, j=1, \cdots, n$ such that $A \in C$ and $\phi \in \mathscr{S}_{\alpha, A}$.

Examples of open convex cones having property $(C)$ are $C=(0, \infty)$, $C=(0, \infty)_{1} \times(0, \infty)_{2} \times \cdots \times(0, \infty)_{n}$, and the forward light cone $C=\Gamma^{+}=$ $\left\{y \in \mathbf{R}^{n}: y_{1}>\left(\sum_{j=2}^{n}\left(y_{j}\right)^{2}\right)^{(1 / 2)}\right\}$. These cones define the upper half plane in $\mathbf{C}^{1}$, the octant formed by the product of $n$ upper half planes in $\mathbf{C}^{n}$, and the tube domain corresponding to the forward light cone $\Gamma^{+}$in $\mathbf{C}^{n}$, respectively; and each of these three domains in complex space is an example of a tubular radial domain. In this paper ( $\$ \$ 4$ and 5) we shall obtain theorems concerning functions analytic in tubular radial domains which are defined by open convex cones which may or may not satisfy property $(\mathrm{C})$. In either case the corresponding theorems for the domain of analyticity being the upper half plane, octant, or tube domain corresponding to the forward light cone will be special cases of the theorems we obtain in $\S \S 4$ and 5 .

The following four lemmas concern the spaces $\mathscr{S}_{\alpha}^{\prime}$, which are the duals of the spaces of type $\mathscr{S}, \mathscr{S}_{\alpha}$.

Lemma 6. Let $V \in \mathscr{D}^{\prime}$. Let $C$ be an open convex cone which satisfies property (C); and let $\left(\exp (-2 \pi\langle y, t\rangle) V_{t}\right) \in \mathscr{S}_{\alpha}^{\prime}$ for every $y \in C$, where $\alpha=\left(\alpha_{1}, \cdots, \alpha_{n}\right), \alpha_{j} \geqq 1, j=1, \cdots, n$. Then $V \in \mathscr{S}_{\alpha}^{\prime}$. 
Proof. Let $\phi \in \mathscr{S}_{\alpha}$. According to property (C), there exists an $n$-tuple $A=\left(A_{1}, \cdots, A_{n}\right), A_{j}>0, j=1, \cdots, n$, such that $A \in C$ and $\phi \in \mathscr{S}_{\alpha, A}$. Since $C$ is a cone then $y=(1 / 2) A \in C$. We now proceed exactly as in the proof of [4, Lemma 2] to obtain the desired result; for we have obtained in our present setting exactly the properties that permit the proof of [4, Lemma 2] to hold. Further the method holds equally well for $\mathscr{S}_{\alpha}^{\prime}, \alpha=\left(\alpha_{1}, \cdots, \alpha_{n}\right), \alpha_{j} \geqq 1, j=1, \cdots, n$.

Lemma 7. Let $V \in \mathscr{S}_{\alpha}^{\prime} \subset \mathscr{D}^{\prime}$ and $\phi \in \mathscr{S}_{x}$, where $\alpha$ is restricted as in Lemma 6. Then $\phi V \in \mathscr{S}^{\prime}$.

Proof. See Constantinescu [4, Lemma 1] and note that the proof holds equally well for $\alpha=\left(\alpha_{1}, \cdots, \alpha_{n}\right), \alpha_{i} \geqq 1, j=1, \cdots, n$.

LeMma 8. Let $C$ be an open convex cone and let y be an arbitrary but fixed point of $C$. Let $\left\{\phi_{n}\right\}$ be a sequence of functions in $\mathscr{S}_{\alpha}$ which converges to zero in $\mathscr{S}_{\alpha}$ as $n \rightarrow \infty$, and put

$$
\psi_{n}(t)=\left(\lambda(t) e^{-2 \pi\langle y, t\rangle} \phi_{n}(t)\right), \quad n=1,2,3,4, \cdots,
$$

where $\lambda(t)$ is the function defined in (17) corresponding to $b \geq 0$. Then $\left\{\psi_{n}\right\}$ is a sequence of functions in $\mathscr{S}_{\alpha}$ which converges to zero in $\mathscr{S}_{\alpha}$ as $n \rightarrow \infty$.

Proof. It is obvious that for each $n, \psi_{n}(t) \in C^{\infty}$. Since $\left\{\phi_{n}\right\}$ converges to zero in $\mathscr{S}_{\alpha}$, there exists an $n$-tuple $A=\left(A_{1}, \cdots, A_{n}\right)$ of positive real numbers such that $\phi_{n} \in \mathscr{S}_{\alpha, A}$ for each $n$ and $\phi_{n} \rightarrow 0$ in $\mathscr{S}_{\alpha, A}$ as $n \rightarrow \infty$. Let $p=2,3,4, \cdots$ be arbitrary but fixed. For the fixed $p$, let $\gamma$ be an arbitrary $n$-tuple of nonnegative integers such that $|\gamma| \leqq$ p. By the generalized Leibnitz rule we have

$$
M_{p}(t)\left|D^{\gamma} \psi_{n}(t)\right| \leqq M_{p}(t) \sum_{\beta+\rho=\gamma} \frac{\gamma !}{\beta ! \rho !}\left|D^{\beta}\left(\lambda(t) e^{-2 \pi\langle y, t)}\right)\right|\left|D_{t}^{\rho}\left(\phi_{n}(t)\right)\right|
$$

$$
\leqq M_{p}(t) \sum_{\beta+\rho=\gamma}\left(\frac{\gamma !}{\beta ! \rho !}\left|D_{t}^{\rho}\left(\phi_{n}(t)\right)\right|\left(\sum_{\nu+\mu=\beta} \frac{\beta !}{\nu ! \mu !}\left|D^{\nu} \lambda(t)\right|\left|y^{\mu} e^{-2 \pi\langle y, t}\right|\right)\right),
$$

where $M_{p}(t)$ is the function defined in (2). Recalling the definition of $\lambda(t)$, we may now argue as in the proof of Lemma 4 and consider (19) for $t \in \mathbf{R}^{n}$ such that $\langle y, t\rangle>(-b-\epsilon), y \in C$. From (19) we thus obtain

$$
M_{p}(t)\left|D^{\gamma} \psi_{n}(t)\right| \leqq \mid \phi_{n} \|_{p} \sum_{\beta+\rho=\gamma} \frac{\gamma !}{\beta ! \rho !}\left(\sum_{\nu+\mu=\beta} \frac{\beta !}{\nu ! \mu !} K_{\nu}\left|y^{\mu}\right| e^{2 \pi(b+\epsilon)}\right)
$$


where $y \in C, b \geqq 0$, and $\epsilon>0$ are fixed and $K_{\nu}$ bounds $\left|D^{\nu} \lambda(t)\right|$. (20) holds for each $\gamma,|\gamma| \leqq p$, and all $t \in \mathbf{R}^{n}$. Since $\left\{\phi_{n}\right\}$ converges to zero in $\mathscr{S}_{x, A}$ then $\left\|\phi_{n}\right\|_{p}$ is uniformly bounded independent of $n$. It now follows from (20) that the same is true for $\left\|\psi_{n}\right\|_{p}$ by an argument similar to obtaining (10), and $p=2,3,4, \cdots$ is arbitrary. It also follows that $\psi_{n}(t) \in \mathscr{S}_{\alpha, A}$ for every $n$.

Now let $\gamma$ be an arbitrary $n$-tuple of nonnegative integers and let $t \in \mathbf{R}^{n}$ be restricted to any interval $\left\{t:\left|t_{j}\right| \leqq d_{j}<\infty, j=1, \cdots, n\right\}$. We still need only consider such $t$ that also satisfy $\langle y, t\rangle>(-b-\epsilon), y \in C$, because of $\lambda(t)$. We have

$$
\left|D^{\gamma} \psi_{n}(t)\right| \leqq \sum_{\beta+\rho=\gamma}\left(\frac{\gamma !}{\beta ! \rho !}\left|D_{t}^{\rho}\left(\phi_{n}(t)\right)\right|\left(\sum_{\nu+\mu=\beta} \frac{\beta !}{\nu ! \mu !}\left|D^{\nu} \lambda(t)\right|\left|y^{\mu}\right| e^{2 \pi(b+\epsilon)}\right)\right)
$$

$$
\leqq e^{2 \pi(b+\epsilon)} \sum_{\beta+\rho=\gamma}\left(\frac{\gamma !}{\beta ! \rho !}\left|D_{t}^{\rho}\left(\phi_{n}(t)\right)\right|\left(\sum_{\nu+\mu=\beta} \frac{\beta !}{\nu ! \mu !} K_{\nu}\left|y^{\mu}\right|\right)\right),
$$

where $y \in C, \quad b \geqq 0, \quad$ and $\epsilon>0$ are fixed, and $K_{v}$ bounds $\left|D^{\nu} \lambda(t)\right|$. Since $\left\{\phi_{n}\right\}$ converges to zero in $\mathscr{S}_{\alpha, A}$, then $\left\{D_{t}^{\rho}\left(\phi_{n}(t)\right)\right\}$ converges to zero uniformly on $\left\{t:\left|t_{j}\right| \leqq d_{j}<\infty, j=1, \cdots, n\right\}$. (21) proves that the same is true of $\left\{D_{t}^{\gamma}\left(\psi_{n}(t)\right)\right\}$. Thus we have proved that $\psi_{n} \in \mathscr{S}_{\alpha, A}$ for each $n$ and $\left\{\psi_{n}\right\}$ converges to zero in $\mathscr{S}_{\alpha, A}$. Thus $\left\{\psi_{n}\right\}$ converges to zero in $\mathscr{S}_{\alpha}$.

The following result is a converse to Lemma 6.

Lemma 9. Let $C$ be an open convex cone. Let $V \in \mathscr{S}_{\alpha}^{\prime}$ with $\operatorname{supp}(V) \subseteq S_{b}=\left\{t: u_{C}(t) \leqq b\right\}$, where $b \geqq 0$ is arbitrary but fixed. Then $\left(\exp (-2 \pi\langle y, t\rangle) V_{t}\right) \in \mathscr{Y}_{\alpha}^{\prime}$ for all $y \in C$.

Proof. Let $\phi \in \mathscr{Y}_{\alpha}$. From the proof of Lemma 4, $(\lambda(t) \exp (-$ $2 \pi\langle y, t\rangle) \phi(t)) \in \mathscr{S}_{\alpha}, y \in C$. Since $\operatorname{supp}(V) \subseteq S_{b}$,

$$
\left\langle e^{-2 \pi\langle y, t\rangle} V, \phi(t)\right\rangle=\left\langle V, \lambda(t) e^{-2 \pi\langle y, t\rangle} \phi(t)\right\rangle .
$$

From the fact that $V \in \mathscr{S}_{\alpha}^{\prime}$ and (22), it is easily seen that (exp($\left.2 \pi\langle y, t\rangle) V_{t}\right)$ is a linear functional on $\mathscr{S}_{\alpha}, y \in C$. Let $\left\{\phi_{n}\right\}$ be a sequence of functions in $\mathscr{S}_{x}$ which converges to zero in $\mathscr{S}_{x}$. By Lemma 8 and the continuity of $V \in \mathscr{Y}_{\alpha}^{\prime}$ we have

$$
\lim _{n \rightarrow \infty}\left\langle e^{-2 \pi\langle y, t\rangle} V, \phi_{n}(t)\right\rangle=\lim _{n \rightarrow \infty}\left\langle V, \psi_{n}(t)\right\rangle=0
$$

where $y$ is arbitrary but fixed in $C$. This proves that (exp ($2 \pi\langle y, t\rangle) V_{t}$ ) is continuous on $\mathscr{S}_{\alpha}$, and the proof is complete. 
4. The space $\boldsymbol{U}_{C}^{b}$ and distributional boundary values in $\left(\mathscr{S}^{x}\right)^{\prime}$. We now define the space of analytic functions $\mathcal{U}_{C}^{b}$ with which we are concerned in this paper. Let $C$ be an open convex cone, and let $C^{\prime}$ be an arbitrary compact subcone of $C$. Let $N(0, m)$ denote a closed ball of the origin in $\mathbf{R}^{n}$ of radius $m>0$. Denote $T\left(C^{\prime}, m\right)=$ $\mathbf{R}^{n}+i\left(C^{\prime} \backslash\left(C^{\prime} \cap N(0, m)\right)\right)$. We consider functions $f(z)$ which satisfy

$$
\begin{gathered}
|f(z)| \leqq K\left(C^{\prime}\right)\left(1+(t z \mid)^{N} \exp (2 \pi(b+\sigma)|y|),\right. \\
z=x+i y \in T\left(C^{\prime}, m\right)
\end{gathered}
$$

for all $\sigma>0$, where $m>0, K\left(C^{\prime}\right)$ is a constant depending on $C^{\prime}, N$ is a nonnegative real number, and $b$ is an arbitrary but fixed nonnegative real number. We denote by $\mathcal{U}_{C}^{b}$ the set of all functions $f(z)$ which are analytic in $T\left(C^{\prime}, m\right)$ and satisfy (23) where $C^{\prime}$ is an arbitrary compact subcone of $C$ and $m>0$ is arbitrary.

We shall now prove two lemmas which we shall need for our study of the functions $\mathcal{U}_{C}^{b}$. In the following two lemmas and throughout the remainder of this paper $T\left(C^{\prime}, m\right)$ denotes the set $\mathbf{R}^{n}+$ $i\left(C^{\prime} \backslash\left(C^{\prime} \cap N(0, m)\right)\right)$.

Lemma 10. Let $C$ be an open convex cone, and let $C^{\prime}$ be an arbitrary compact subcone of $C$. Let $g(t), t \in \mathbf{R}^{n}$, be a continuous function with support in $C^{*}=S_{0}=\left\{t: u_{C}(t) \leqq 0\right\}$. Let

$$
|g(t)| \leqq M\left(C^{\prime}\right) \exp (2 \pi(\langle\Omega, t\rangle+\sigma|\Omega|)), \quad t \in \mathbf{R}^{n},
$$

for all $\sigma>0$, where $M\left(C^{\prime}\right)$ is a constant depending on $C^{\prime} \subset C$ and (24) is independent of $\Omega \in\left(C^{\prime} \backslash\left(C^{\prime} \cap N(0, m)\right)\right)$, where $m>0$ is arbitrary but fixed (i.e., (24) holds for all $\Omega \in\left(C^{\prime} \backslash\left(C^{\prime} \cap N(0, m)\right)\right)$.) Let $y$ be an arbitrary but fixed point of $\left(C^{\prime} \backslash\left(C^{\prime} \cap N(0, m)\right)\right)$. Then $(\exp (-$ $2 \pi\langle y, t\rangle) g(t)) \in L^{p}, 1 \leqq p<\infty$, as a function of $t \in \mathbf{R}^{n}$.

Proof. Let $y$ be an arbitrary but fixed point of $\left(C^{\prime} \backslash\left(C^{\prime} \cap N(0, m)\right)\right)$ where $m>0$ is arbitrary but fixed. We choose $\Omega$ in (24) as $\Omega=\mu y$ where $\mu$ is a positive real number such that $1>\mu>(m /|y|)>0$. (For $y \in\left(C^{\prime} \backslash\left(C^{\prime} \cap N(0, m)\right)\right),|y|>m$. Thus $1>(m /|y|)>0$, and $(m /|y|)$ is fixed since both $m>0$ and $y$ are fixed. We thus choose a real number $\mu$ such that $1>\mu>(m /|y|)>0$. Since $C^{\prime}$ is a cone and $y \in C^{\prime}$, then $\mu y \in C^{\prime}$. Thus $\Omega=\mu y \in\left(C^{\prime} \backslash\left(C^{\prime} \cap N(0, m)\right)\right)$ since $\mu y \in C^{\prime}$ and $|\mu y|=\mu|y|>m$. By assumption, (24) holds for this particular choice of $\Omega$.) By Vladimirov [14, Lemma 2, p. 223] there exists a real number $d>0$ such that 


$$
\langle t, y\rangle \geqq d|y||t|
$$

for all $t \in C^{*}$ and $y \in C^{\prime} \subset C$. Using the above choice of $\Omega$, we have from (24) and (25) that

$$
\left|e^{-2 \pi\langle y, t\rangle} g(t)\right| \leqq M\left(C^{\prime}\right) \exp (2 \pi \sigma \mu|y|) \exp (2 \pi(1-\mu)(-\langle y, t\rangle))
$$

$$
\leqq M\left(C^{\prime}\right) \exp (2 \pi \sigma \mu|y|) \exp (-2 \pi(1-\mu) d|y||t|) ;
$$

and (26) holds for all $t \in C^{*}$ and $y \in\left(C^{\prime} \backslash\left(C^{\prime} \cap N(0, m)\right)\right)$. From the choice of $\mu,(1-\mu)>0$; and from $(25), d>0$. Now let $1 \leqq p<\infty$ and recall that $\operatorname{supp}(g) \subseteq C^{*}$. We have from (26) that

$$
\int_{\mathbf{R}^{n}}\left|e^{-2 \pi\langle y, t\rangle} g(t)\right|^{p} d t=\int_{C^{*}}\left|e^{-2 \pi\langle y, t\rangle} g(t)\right|^{p} d t
$$

$$
\leqq\left(M\left(C^{\prime}\right)\right)^{p} \exp (2 \pi \sigma \mu p|y|) \int_{C^{*}} \exp (-2 \pi(1-\mu) d p|y||t|) d t .
$$

Using a classical result concerning the Lebesgue integral (see Schwartz [13, Theorem 32, p. 39]) and (27) we have

(28)

$$
\int_{\mathbf{R}^{n}}\left|e^{-2 \pi(y, t)} g(t)\right|^{p} d t
$$

$$
\leqq\left(M\left(C^{\prime}\right)\right)^{p}\left(Z_{n}\right) \exp (2 \pi \sigma \mu p|y|) \int_{0}^{\infty} s^{n-1} \exp (-2 \pi(1-\mu) d p|y| s) d s,
$$

where $Z_{n}$ is the area of the unit sphere in $\mathbf{R}^{n}$; and $(1-\mu)>0$ and $d>0$. Integrating by parts $(n-1)$ times on the last integral in (28) we obtain

$$
\int_{\mathbf{R}^{n}}\left|e^{-2 \pi\langle y, t} g(t)\right|^{p} d t
$$

$$
\leqq\left(M\left(C^{\prime}\right)\right)^{p}\left(Z_{n}\right) \exp (2 \pi \sigma \mu p|y|)(n-1) !(2 \pi(1-\mu) d p|y|)^{-n} .
$$

Now (29) holds for all $\sigma>0, y$ is fixed in $\left(C^{\prime} \backslash\left(C^{\prime} \cap N(0, m)\right)\right)$, and $\mu$ is fixed. Thus the right hand side of (29) is finite; and we conclude from (29) that $(\exp (-2 \pi\langle y, t\rangle) g(t)) \in L^{p}, 1 \leqq p<\infty$, as a function of $t \in \mathbf{R}^{n}$ for fixed $y \in\left(C^{\prime} \backslash\left(C^{\prime} \cap N(0, m)\right)\right)$.

Lemma 11. Let $C$ be an open convex cone, and let $C^{\prime}$ be an arbitrary compact subcone of $C$. Let $V=D^{\gamma} g(t)$, where $g(t)$ is a 
continuous function on $\mathbf{R}^{n}$ which satisfies (24). Let $\operatorname{supp}(V) \subseteq C^{*}=$ $S_{0}$. Then $f(z)=\langle V, \exp (2 \pi i\langle z, t\rangle)\rangle$ is an element of $\mathcal{U}_{c}^{0}$.

Proof. Let $C^{\prime}$ be an arbitrary compact subcone of $C$ and $m>0$ be arbitrary but fixed. $C^{*}$ is a regular set (Schwartz [12, pp. 98-99]); thus $\operatorname{supp}(g)=\operatorname{supp}(V) \subseteq C^{*}$. We now consider the function

$$
f(z)=\left\langle V, e^{2 \pi i(z, t)}\right\rangle=(-1)^{|\gamma|} z^{\gamma} \int_{C^{*}} g(t) e^{2 \pi i\langle z, t\rangle} d t .
$$

(With $V=D^{\gamma} g(t)$, we have formally used the definition of distributional derivative in (30). By what we prove below, this formalism is valid.) We wish to prove the existence and analyticity of $f(z)$ for a suitable restriction of $y=\operatorname{Im}(z)$. To do so it suffices to consider

$$
h(z)=\int_{C^{*}} g(t) e^{2 \pi i\langle z, t\rangle} d t
$$

Let $z_{0}$ be an arbitrary but fixed point of $T\left(C^{\prime}, m\right)$ and let $R\left(z_{0}, r\right) \subset$ $T\left(C^{\prime}, m\right)$ be an arbitrary but fixed neighborhood of $z_{0}$ with radius $r$ whose closure is in $T\left(C^{\prime}, m\right)$. Let $z \in R\left(z_{0}, r\right)$, and let $\beta$ be an arbitrary $n$-tuple of nonnegative integers. From Vladimirov [14, Lemma 2, p. 223] we obtain the existence of a real number $d>0$ such that

$$
\langle t, y\rangle \geqq d|t|, z=x+i y \in R\left(z_{0}, r\right),
$$

for all $t \in C^{*}$. Since $R\left(z_{0}, r\right)$ is fixed and has closure in $T\left(C^{\prime}, m\right)$, there exist two balls of the origin in $\mathbf{R}^{n}$ of radius $S$ and $T$, respectively, such that

$$
0<m<S<|y|<T
$$

for all $y=\operatorname{Im}(z), z=x+i y \in R\left(z_{0}, r\right)$. We now let $\mu=(m / S)$. Then $0<\mu<1$. Further, $\mu y \in C^{\prime}$ for $y=\operatorname{Im}(z), z=x+i y \in R\left(z_{0}, r\right)$, since $C^{\prime}$ is a cone; and $|\mu y|=\mu|y|=(m / S)|y|>(m / S)(S)=m$. Thus if $\mu=(m / S) \quad$ and $\quad y=\operatorname{Im}(z), \quad z=x+i y \in R\left(z_{0}, r\right)$, then $\mu y \in\left(C^{\prime} \backslash\left(C^{\prime} \cap N(0, m)\right)\right)$. We now choose $\Omega=\mu y, \mu=(m / S), y=$ $\operatorname{Im}(z), z=x+i y \in R\left(z_{0}, r\right)$, in (24). With this choice of $\Omega$, we have from (24), (32), and the fact that $|y|<T$ for all $y=\operatorname{Im}(z), z=$ $x+i y \in R\left(z_{0}, r\right)$, that 


$$
\begin{aligned}
& \left|\int_{C^{*}} g(t) t^{\beta} e^{2 \pi i(z, t)} d t\right| \leqq \int_{C^{*}}\left|t^{\beta}\right||g(t)| e^{-2 \pi(y, t)} d t \\
& \quad \leqq M\left(C^{\prime}\right) \int_{C^{*}}\left|t^{\beta}\right| e^{2 \pi \mu\langle y, t\rangle} e^{2 \pi \sigma \mu|y|} e^{-2 \pi\langle y, t\rangle} d t \\
& \quad \leqq M\left(C^{\prime}\right) e^{2 \pi \sigma \mu T} \int_{C^{*}}\left|t^{\beta}\right| \exp (-2 \pi(1-\mu) d|t|) d t
\end{aligned}
$$

and $(1-\mu)>0, d>0$. Again using the result in Schwartz [13, Theorem 32, p. 39] as in (28), we have from (33) that

$$
\begin{aligned}
& \left|\int_{C^{*}} g(t) t^{\beta} e^{2 \pi i(z, t)} d t\right| \\
& \leqq M\left(C^{\prime}\right)\left(Z_{n}\right) e^{2 \pi \sigma \mu T} \int_{0}^{\infty} s^{|\beta|+n-1} \exp (-2 \pi d(1-\mu) s) d s \\
& <\infty
\end{aligned}
$$

where as in (28) $Z_{n}$ denotes the area of the unit sphere in $\mathbf{R}^{n}$. From (34) we conclude that the integral defining $h(z)$ in (31) and any derivative, $D_{z}^{\beta} h(z)$, of it converges uniformly for $z \in R\left(z_{0}, r\right)$. (Recall that $\mu=(m / S), \sigma>0$, and $T$ are all independent of $z \in R\left(z_{0}, r\right)$.) Since $z_{0}$ is an arbitrary point in $T\left(C^{\prime}, m\right)$, we thus have that $h(z)$ defined in (31) exists and is analytic for $z \in T\left(C^{\prime}, m\right)$. From (30) it thus follows that $f(z)$ exists and is analytic for $z \in T\left(C^{\prime}, m\right)$, where $C^{\prime}$ is an arbitrary compact subcone of $C$ and $m>0$ is arbitrary.

Letting $\beta=(0, \cdots, 0)$ in (34), we have immediately from (30) and (34) the existence of a constant $K\left(C^{\prime}\right)$ and a positive real number $N$ such that

$$
|f(z)| \leqq\left|z^{\gamma}\right||h(z)| \leqq K\left(C^{\prime}\right)(1+|z|)^{N}, z \in T\left(C^{\prime}, m\right) .
$$

(Note that $K\left(C^{\prime}\right)$ also depends on $V$ here because of the $\gamma$ in $z^{\gamma}$.) But for any $\sigma>0$ and $y \in\left(C^{\prime} \backslash\left(C^{\prime} \cap N(0, m)\right)\right), \exp (2 \pi \sigma|y|)>1$. Thus

$$
|f(z)| \leqq K\left(C^{\prime}\right)(1+|z|)^{N} \exp (2 \pi \sigma|y|), \quad z=x+i y \in T\left(C^{\prime}, m\right) .
$$

This completes the proof that $f(z)=\langle V, \exp (2 \pi i\langle z, t\rangle)\rangle \in \mathcal{U}_{c}^{0}$.

Throughout the remainder of this section we assume that $C$ is an open convex cone such that property (C) (see \$3) is satisfied by each compact subcone $C^{\prime}$ of $C$, and $C^{\prime}$ will always denote such a compact subcone of $C$. Further, we assume throughout the remainder of this section that the $n$-tuple $\alpha=\left(\alpha_{1}, \cdots, \alpha_{n}\right)$ is such that $\alpha_{j} \geqq 1, j=1, \cdots, n$. 
In the following theorem we show that elements of the space of analytic functions $\mathcal{U}_{C}^{b}$ obtain distributional boundary values in $\left(\mathscr{S}^{\alpha}\right)^{\prime}$ on the distinguished boundary of the tubular radial domain $T^{C}$.

THEOREM 1. Let $f(z) \in \mathcal{U}_{C}^{b}$, where $C$ is an open convex cone such that property $(\mathrm{C})$ is satisfied by each compact subcone $C^{\prime} \subset C$. Then there exists a unique element $V \in \mathscr{S}_{\alpha}^{\prime}$ with $\operatorname{supp}(V) \subseteq S_{b}=$ $\left\{t: u_{C}(t) \leqq b\right\}$ such that $\left(\exp (-2 \pi\langle y, t\rangle) V_{t}\right) \in \mathscr{S}_{\alpha}^{\prime}$ for all $y \in C$ and $f(z) \rightarrow \mathscr{F}[V] \in\left(\mathscr{S}^{x}\right)^{\prime}$ in the weak topology of $\left(\mathscr{S}^{x}\right)^{\prime}$ as $y \rightarrow 0, y \in C^{\prime} \subset C$.

Proof. Let $C^{\prime}$ be an arbitrary but fixed compact subcone of $C$. By hypothesis $f(z) \in \mathcal{U}_{C}^{b}$; thus for any $m>0 f(z)$ is analytic in $T\left(C^{\prime}, m\right)=\mathbf{R}^{n}+i\left(C^{\prime} \backslash\left(C^{\prime} \cap N(0, m)\right)\right)$ and satisfies (23) there. We may choose an $n$-tuple $W=\left(w_{1}, \cdots, w_{n}\right)$ of nonnegative integers such that

$$
\begin{gathered}
\left|z^{-w} f(z)\right| \leqq K\left(C^{\prime}\right)(1+|z|)^{-n-\epsilon} \exp (2 \pi(b+\sigma)|y|) \\
z=x+i y \in T\left(C^{\prime}, m\right)
\end{gathered}
$$

where $n$ is the dimension and $\epsilon>0$ is fixed. Put

$$
\mathrm{g}_{\mathrm{y}}(\mathrm{t})=\int_{\mathbf{R}^{n}} z^{-\mathrm{w}} f(z) e^{-2 \pi i(z, t)} d x, z=x+i y \in T\left(C^{\prime}, m\right) .
$$

Because of (35), $g_{y}(t)$ exists and is a continuous function of $t \in \mathbf{R}^{n}$. We may now apply exactly the same method of proof as in Carmichael [2, Theorem 1] to show that $g_{y}(t)$ is independent of $y=\operatorname{Im}(z)$ and $\operatorname{supp}\left(g_{y}\right) \subseteq S_{b}=\left\{t: u_{C}(t) \leqq b\right\}$. (From now on we write $g(t)$ instead of $g_{y}(t)$ to emphasize the independence of the function defined in (36) from $y=\operatorname{Im}(z)$.) From (35) we see that $\left(z^{-w} f(z)\right) \in L^{1} \cap L^{2}$ as a function of $x=\operatorname{Re}(z) \in \mathbf{R}^{n}, \quad y \in\left(C^{\prime} \backslash\left(C^{\prime} \cap N(0, m)\right)\right)$. Thus from (36) and the Plancherel theory. we have $(\exp (-2 \pi\langle y, t\rangle) g(t)) \in L^{2}$ as a function of $t \in \mathbf{R}^{n}$; and

$$
z^{-w} f(z)=\mathscr{F}\left[e^{-2 \pi\langle y, t\rangle} g(t): x\right], \quad z=x+i y \in T\left(C^{\prime}, m\right),
$$

where the Fourier transform is in the $L^{2}$ sense. From (35) and (36) we have

$$
|g(t)| \leqq K\left(C^{\prime}\right) \exp (2 \pi(\langle y, t\rangle+(b+\sigma)|y|)) \int_{\mathbf{R}^{n}}(1+|z|)^{-n-\epsilon} d x
$$

$$
\leqq M\left(C^{\prime}\right) \exp (2 \pi(\langle y, t\rangle+(b+\sigma)|y|))
$$


$z=x+i y \in T\left(C^{\prime}, m\right)$, for all $\sigma>0$, where $M\left(C^{\prime}\right)$ is a constant depending on $C^{\prime} \subset C$ and the boundedness property (38) is independent of $y \in\left(C^{\prime} \backslash\left(C^{\prime} \cap N(0, m)\right)\right)$ since $g(t)$ is independent of $y$. Thus from (38) we have

$$
\left|e^{-2 \pi\langle y, t\rangle} g(t)\right| \leqq M\left(C^{\prime}\right) e^{(b+\sigma)|y|}, y \in\left(C^{\prime} \backslash\left(C^{\prime} \cap N(0, m)\right)\right)
$$

Hence for $y$ arbitrary but fixed in $\left(C^{\prime} \backslash\left(C^{\prime} \cap N(0, m)\right)\right),(\exp (-2 \pi\langle y, t\rangle)$ $g(t))$ is a continuous bounded function of $t \in \mathbf{R}^{n}$; so that $(\exp (-2 \pi\langle y, t\rangle) \quad g(t)) \in \mathscr{S}^{\prime} \subset \mathscr{S}_{\alpha}^{\prime}$. Further $g(t) \in \mathscr{D}^{\prime}$ since it is continuous. Applying Lemma 6 and its proof, we have $g(t) \in$ $\mathscr{S}_{\alpha}^{\prime}$. (We may invoke Lemma 6 here because the exact same proof as in Lemma 6 will give the desired result that $g(t) \in \mathscr{S}_{\alpha}^{\prime}$ under the assumptions that $g(t) \in \mathscr{D}^{\prime}, \quad(\exp (-2 \pi\langle y, t\rangle) g(t)) \in \mathscr{S}_{\alpha}^{\prime}$ for all $y \in$ $\left(C^{\prime} \backslash\left(C^{\prime} \cap N(0, m)\right)\right)$, and $C^{\prime}$ satisfies property $(C)$, which we have assumed for all compact subcones $C^{\prime}$ in this theorem. This is true for since $C^{\prime}$ is an open convex cone itself and has property $(C)$, then for each $\phi \in \mathscr{S}_{\alpha}$ there exists an $n$-tuple $A=\left(A_{1}, \cdots, A_{n}\right), A_{l}>0, j=$ $1, \cdots, n$, such that $A \in C^{\prime}$ and $\phi \in \mathscr{S}_{\alpha, A}$. Now if $A \in$ $\left(C^{\prime} \backslash\left(C^{\prime} \cap N(0, m)\right)\right)$ then let $D=A$; while if $A \notin\left(C^{\prime} \backslash\left(C^{\prime} \cap N(0, m)\right)\right)$, choose $\xi>0$ large enough so that $D=$ $\xi A \in\left(C^{\prime} \backslash\left(C^{\prime} \cap N(0, m)\right)\right)$. This may be done since $C^{\prime}$ is a cone. In either case $\phi \in \mathscr{S}_{\alpha, D}$ since $A_{j} \leqq D_{j}, \quad j=1, \cdots, n$, implies $\mathscr{S}_{\alpha, A} \subseteq$ $\mathscr{S}_{x, D}$. Thus we now have an $n$-tuple $D$ of positive real numbers such that $D \in\left(C^{\prime} \backslash\left(C^{\prime} \cap N(0, m)\right)\right)$ and $\phi \in \mathscr{S}_{r . D}$. Now making the choice of $y=\mu D \in\left(C^{\prime} \backslash\left(C^{\prime} \cap N(0, m)\right)\right)$, where $\mu>0$ such that $1>\mu>$ $(m /|D|)>0$ (recall the proof of Lemma 10 where we chose $\Omega=\mu y$ ), we may proceed as indicated in the proof of Lemma 6 to obtain the result that $g(t) \in \mathscr{S}_{\alpha}^{\prime}$. Note here that the selection of $y=$ $\mu D \in\left(C^{\prime} \backslash\left(C^{\prime} \cap N(0, m)\right)\right)$ is the same step as letting $y=(1 / 2) A$ in the proof of Lemma 6.$)$

We now define the differential operator $\Delta$ by

$$
\Delta=((-1) /(2 \pi i))^{|w|} \frac{\partial^{|\omega|}}{\partial t_{1}^{w_{1}} \cdots \partial t_{n}^{w_{n}}},
$$

where $W$ is the $n$-tuple chosen at the beginning of this proof. Since $g(t) \in \mathscr{Y}_{\alpha}^{\prime}$,

$$
V=\Delta g(t) \in \mathscr{S}_{\alpha}^{\prime} .
$$

Further, $\operatorname{supp}(V)=\operatorname{supp}(g) \subseteq S_{b}=\left\{t: u_{C}(t) \leqq b\right\} . \quad$ Thus by Lemma 9 , $\left(\exp (-2 \pi\langle y, t\rangle) V_{t}\right) \in \mathscr{S}_{\alpha}^{\prime}$ for all $y \in C$.

It remains to prove the desired convergence. . Foe the moment we let $y$ be an arbitrary but fixed point of $\left(C^{\prime} \backslash\left(C^{\prime} \cap N(0, m)\right)\right)$. As we 
have shown above, $(\exp (-2 \pi\langle y, t\rangle) g(t)) \in \mathscr{Y}_{\alpha}^{\prime}, \quad y \in$ $\left(C^{\prime} \backslash\left(C^{\prime} \cap N(0, m)\right)\right)$ and $\left(z^{-w} f(z)\right) \in L^{1} \cap L^{2}$ as a function of $x \in \mathbf{R}^{n}, y \in$ $\left(C^{\prime} \backslash\left(C^{\prime} \cap N(0, m)\right)\right)$. Thus (37) holds as an equality in $\left(\mathscr{S}^{\alpha}\right)^{\prime}$; and from (4) we have

$$
\begin{aligned}
\left\langle z^{-w} f(z), \psi(x)\right\rangle & =\left\langle\mathscr{F}\left[e^{-2 \pi(y, t)} g(t) ; x\right], \psi(x)\right\rangle \\
& =\left\langle e^{-2 \pi\langle y, t\rangle} g(t), \phi(t)\right\rangle,
\end{aligned}
$$

$z=x+i y \in T\left(C^{\prime}, m\right)$, for $y$ arbitrary but fixed in $\left(C^{\prime} \backslash\left(C^{\prime} \cap N(0, m)\right)\right)$, where $\phi \in \mathscr{S}_{x}$ and $\psi=\hat{\phi} \in \mathscr{S}^{x}$. Since $\operatorname{supp}(g) \subseteq S_{b}$, we have

$$
\left\langle e^{-2 \pi\langle y, t\rangle} g(t), \phi(t)\right\rangle=\left\langle g(t), \lambda(t) e^{-2 \pi(y, t)} \phi(t)\right\rangle,
$$

where $\lambda(t)$ is the function defined in (17). By Lemma 4, $(\lambda(t)$ $\exp (-2 \pi\langle y, t\rangle) \phi(t)) \rightarrow(\lambda(t) \phi(t))$ in $\mathscr{S}_{\alpha}$ as $y \rightarrow 0, y \in C^{\prime} \subset C$. Since $g(t) \in \mathscr{S}_{\alpha}^{\prime}$ and $\operatorname{supp}(g) \subseteq S_{b}$, we thus have

$$
\lim _{\substack{y \rightarrow 0 \\ y \in C^{\prime} \subset C}}\left\langle g(t), \lambda(t) e^{-2 \pi\langle y, t\rangle} \phi(t)\right\rangle=\langle g(t), \lambda(t) \phi(t)\rangle=\langle g(t), \phi(t)\rangle .
$$

Combining (40), (41), and (42) and using (4), we obtain (recall $m>0$ is arbitrary)

$$
\lim _{\substack{y \rightarrow 0 \\ y \in C^{\prime} \subset C}}\left\langle z^{-w} f(z), \psi(x)\right\rangle=\langle g(t), \phi(t)\rangle=\langle\mathscr{F}[g], \psi(x)\rangle ;
$$

from which it follows that

$$
\begin{gathered}
\langle f(z), \psi(x)\rangle=\left\langle z^{-w} f(z), z^{w} \psi(x)\right\rangle \\
\rightarrow\left\langle\mathscr{F}[g], x^{w} \psi(x)\right\rangle=\left\langle x^{w} \mathscr{F}[g], \psi(x)\right\rangle
\end{gathered}
$$

as $y=\operatorname{Im}(z) \rightarrow 0, y \in C^{\prime} \subset C$.

Since $V=\Delta g(t) \in \mathscr{S}_{\alpha}^{\prime}$, then $\mathscr{F}[V] \in\left(\mathscr{S}^{x}\right)^{\prime}$. Letting $\psi(x) \in \mathscr{S}^{x}$, there exists an element $\check{\chi}(t)=\chi(-t) \in \mathscr{S}_{x}$ such that $\psi(x)=\mathscr{F}[\check{\chi}(t): x]$ (recall that the Fourier transform is a continuous linear one-one mapping of $\mathscr{S}_{x}$ onto $\left.\mathscr{S}^{x}\right)$; and this $\check{\chi}(t)$ is given in terms of $\psi(x)$ by

$$
\check{\chi}(t)=\mathscr{F}^{-1}[\psi(x) ;(-t)]=\int_{\mathbf{R}^{n}} \psi(x) e^{2 \pi i\langle x, t\rangle} d x .
$$

By (4) and the definitions of $\Delta$ and of the distributional derivative, 


$$
\langle\widetilde{F}[V], \psi(x)\rangle=\langle V, \check{\chi}(t)\rangle=\left\langle\Delta g(t), \int_{\mathbf{R}^{n}} \psi(x) e^{2 \pi i(x, t)} d x\right\rangle
$$

$$
=\left\langle g(t), \int_{\mathbf{R}^{n}} x^{w} \psi(x) e^{2 \pi i\langle x, t\rangle} d x\right\rangle
$$

It is known that multiplication by a polynomial is a continuous operation in $\mathscr{S}^{x}$ [9, Chapter IV, §4]. Thus $\psi(x) \in \mathscr{S}^{x}$ implies $\left(x^{w} \psi(x)\right) \in \mathscr{S}^{x}$; so that there exists a function $\check{\Gamma}(t)=\Gamma(-t) \in \mathscr{S}_{x}$ for which $x{ }^{w} \psi(x)=$ $\mathscr{F}[\check{\Gamma}(t) ; x]$ and $\check{\Gamma}(t)=\mathscr{F}^{-1}\left[x^{w} \psi(x) ;(-t)\right]$. Thus from (44) and (4) we have

$$
\langle\widetilde{F}[V], \psi(x)\rangle=\langle g(t), \check{\Gamma}(t)\rangle=\left\langle\mathscr{F}[g], x^{w} \psi(x)\right\rangle=\left\langle x^{w} \mathscr{F}[g], \psi(x)\right\rangle,
$$

which shows that $\mathscr{F}[V]=\left(x^{w} \mathscr{F}[g]\right)$ in $\left(\mathscr{S}^{x}\right)^{\prime}$. Combining (43) and (45) we have proved $f(z) \rightarrow \mathscr{F}[V] \in\left(\mathscr{S}^{x}\right)^{\prime}$ as $y=\operatorname{Im}(z) \rightarrow 0, y \in C^{\prime} \subset C$, in the weak topology of $\left(\mathscr{S}^{x}\right)^{\prime}$, which is the desired result. The proof of Theorem 1 is complete.

The space of analytic functions $G_{C}^{b}$ considered in [2, Theorem 1] restricted to cones $C$ under consideration in this section is a subspace of the space $\mathcal{U}_{C}^{b}$ defined in this paper. Now $\left(\mathscr{S}^{x}\right)^{\prime} \subset \mathscr{Z}^{\prime}$, the space of ultra-distributions of Gel'fand and Shilov [8]; and the injection is continuous. We thus see that Theorem 1 of the present paper gives more precise information concerning the distributional boundary values of functions which are analytic in tubular radial domains and which have growth condition as in (23) (corresponding to open convex cones $C$ whose compact subcones $C^{\prime}$ satisfy property $(C)$ ) than was given in [2, Theorem 1]. Further, using similar techniques as in the proof of Theorem 1, [2, Theorem 1] can be restated and proved for the space of functions $U_{C}^{b}$ defined as in this section for $\mathrm{C}$ being an arbitrary open convex cone. Since $G_{C}^{b} \subseteq \mathcal{U}_{C}^{b}$, one obtains a more general result than [2, Theorem 1] for the distributional boundary values in $\mathscr{Z}^{\prime}$.

For the special case that $b=0$, we shall now show that more information can be obtained concerning the functions $f(z) \in \mathcal{U}_{c}^{0}$ than was given in Theorem 1. We shall show that $f(z) \in \mathcal{U}_{C}^{0}$ can be recovered as the Fourier-Laplace transform of the constructed distribution $V \in \mathscr{S}_{\alpha}^{\prime}$ and also as the Fourier transform in $\left(\mathscr{S}^{\alpha}\right)^{\prime}$ of $(\exp (-$ $\left.2 \pi\langle y, t\rangle) V_{t}\right)$. Further, we show that the function $f(z)$ satisfies a strong boundedness property in $\left(\mathscr{S}^{\alpha}\right)^{\prime}$. In the following theorem $m>0$ is arbitrary.

THEOREM 2. Let $f(z) \in U_{C}^{0}$, where $C$ is an open convex cone such that property $(C)$ is satisfied by each compact subcone $C^{\prime} \subset C$. Then there exists a unique element $V \in \mathscr{S}_{\alpha}^{\prime}$ with $\operatorname{supp}(V) \subseteq C^{*}=S_{0}$ such that 


$$
\begin{gathered}
\left(\operatorname { e x p } \left(-2 \pi\langle y, t\rangle\left(V_{t}\right) \in \mathscr{P}_{\alpha}^{\prime} \text { for all } y \in C ;\right.\right. \\
f(z)=\left\langle V, e^{2 \pi i\langle z . t\rangle}\right\rangle, z \in T\left(C^{\prime}, m\right) ; \\
f(z)=\mathscr{F}\left[e^{-2 \pi\langle y . t\rangle} V_{t}\right], z=x+i y \in T\left(C^{\prime}, m\right) \\
\text { where (48) holds as an equality in }\left(\mathscr{S}^{\alpha}\right)^{\prime} ;
\end{gathered}
$$

(49) $\left\{f(z): y=\operatorname{Im}(z) \in\left(C^{\prime} \backslash\left(C^{\prime} \cap N(0, m)\right)\right),|y| \leqq Q\right\}$ is a strongly bounded set in $\left(\mathscr{P}^{\alpha}\right)^{\prime}$, where $Q$ is an arbitrary but fixed positive real number greater than $m$;

(50) $f(z) \rightarrow \mathscr{F}[V] \in\left(\mathscr{P}^{\alpha}\right)^{\prime}$ in the weak topology of $\left(\mathscr{P}^{\alpha}\right)^{\prime}$ as $y=$ $\operatorname{Im}(z) \rightarrow 0, y \in C^{\prime} \subset C$.

Proof. The existence of $V \in \mathscr{S}_{\alpha}^{\prime}$ with $\operatorname{supp}(V) \subseteq C^{*}$ such that (46) and (50) hold follows from Theorem 1. We now prove (47). Recall from the proof of Theorem 1 that $V=\Delta g(t)$, where $g(t)$ is a continuous function with supp $(g) \subseteq C^{*}$ and $g(t)$ grows as in (38) where $b=0$ (i.e. grows as in (24).) Thus by Lemma $11,\langle V, \exp (2 \pi i\langle z, t\rangle)\rangle$ exists and is an analytic function of $z \in T\left(C^{\prime}, m\right)$. Using the definition of $\Delta$ given in the proof of Theorem 1 and a straightforward calculation, we have

$$
\left\langle V, e^{2 \pi \imath\langle z . t\rangle}\right\rangle=z^{w} \mathscr{F}\left[e^{-2 \pi\langle y . t} g(t) ; x\right], \quad z=x+i y \in T\left(C^{\prime}, m\right),
$$

where the Fourier transform in (51) can now be interpreted in both the $L^{1}$ and $L^{2}$ sense because (exp $\left.(-2 \pi\langle y, t\rangle) g(t)\right) \in L^{1} \cap L^{2}$ according to Lemma 10. Combining (37) and (51) we have (47).

In order to prove (48) we let $\psi \in \mathscr{S}^{\alpha}$. There exists an element $\check{\chi}(t)=\chi(-t) \in \mathscr{Y}_{\alpha} \quad$ such that $\quad \psi(x)=\mathscr{F}[\check{\chi}(t) ; x]$ and $\check{\chi}(t)=$ $\mathscr{F}^{-1}[\psi(x) ;(-t)]$ (recall the proof of Theorem 1.) Let $y$ be an arbitrary but fixed point of $\left(C^{\prime} \backslash\left(C^{\prime} \cap N(0, m)\right)\right)$. We have

$$
e^{-2 \pi(y, t)} \check{\chi}(t)=\int_{\mathbf{R}^{n}} \psi(x) e^{2 \pi \iota\langle z, t\rangle} d t, \quad z=x+i y,
$$

and $(\exp (-2 \pi\langle y, t\rangle) \check{\chi}(t)) \in \mathscr{S}_{x}$ since $\check{\chi}(t) \in \mathscr{S}_{x}$. Now $f(z) \in\left(\mathscr{S}^{x}\right)^{\prime}$ as a function of $x=\operatorname{Re}(z) \in \mathbf{R}^{n}$ for $y$ arbitrary but fixed in $\left(C^{\prime} \backslash\left(C^{\prime} \cap\right.\right.$ $N(0, m))$ ). Using (47), a change of order of integration, and (52) we obtain 


$$
\begin{aligned}
\langle f(z), \psi(x)\rangle= & \left\langle\left\langle\Delta g(t), e^{2 \pi i\langle z, t\rangle}\right\rangle, \quad \psi(x)\right\rangle \\
= & \int_{\mathbf{R}^{n}} \psi(x) z^{w} \int_{C^{*}} g(t) e^{2 \pi i\langle z, t\rangle} d t d x \\
= & \int_{C^{*}} g(t) \int_{\mathbf{R}^{n}} z^{w} \psi(x) e^{2 \pi i\langle z, t\rangle} d x d t \\
= & \left\langle V, \int_{\mathbf{R}^{n}} \psi(x) e^{2 \pi i\langle z, t\rangle} d x\right\rangle \\
= & \left\langle e^{-2 \pi\langle y, t\rangle} V, \tilde{\chi}(t)\right\rangle ;
\end{aligned}
$$

and we have by (46) that $\left(\exp (-2 \pi\langle y, t\rangle) V_{t}\right) \in \mathscr{S}_{\alpha}^{\prime}$. From (53), (4), and the fact that $\psi(x)=\mathscr{F}[\check{\chi}(t) ; x]$ we obtain

$$
\langle f(z), \psi(x)\rangle=\left\langle\mathscr{F}\left[e^{-2 \pi(y, t)} V\right], \psi(x)\right\rangle, \psi \in \mathscr{S}^{x},
$$

which proves (48).

We now prove (49). Let $\Phi$ be an arbitrary bounded set in $\mathscr{S}_{\alpha}$; then $\check{\Phi}=\{\check{\phi}(t)=\phi(-t): \phi \in \Phi\}$ is a bounded set in $\mathscr{S}_{\alpha}$. Let $\check{\phi} \in \check{\Phi}$. Since $\operatorname{supp}(V) \subseteq C^{*}$, we have

$$
\left\langle e^{-2 \pi\langle y, t\rangle} V, \check{\phi}(t)\right\rangle=\left\langle V, \lambda(t) e^{-2 \pi(y, t)} \check{\phi}(t)\right\rangle,
$$

where $\lambda(t)$ is defined as in (17) corresponding to $b=0$. By Lemma 5 , $\left\{(\lambda(t) \exp (-2 \pi\langle y, t\rangle) \check{\phi}(t)): \phi \in \Phi, y \in\left(C^{\prime} \backslash\left(C^{\prime} \cap N(0, m)\right)\right),|y| \leqq Q\right\}$ is a bounded set in $\mathscr{S}_{\alpha}$, where $Q$ is an arbitrary but fixed real number greater than $m$. Since $V \in \mathscr{S}_{\alpha}^{\prime}, V$ is continuous and hence bounded on $\mathscr{S}_{\alpha}$. Thus

$$
\begin{aligned}
& \left\{\left\langle e^{-2 \pi(y, t)} V, \check{\phi}(t)\right\rangle: \phi \in \Phi, y \in\left(C^{\prime} \backslash\left(C^{\prime} \cap N(0, m)\right)\right),|y| \leqq Q\right\} \\
& \quad=\left\{\left\langle V, \lambda(t) e^{-2 \pi(y, t)} \check{\phi}(t)\right\rangle: \phi \in \Phi, \quad y \in\left(C^{\prime} \backslash\left(C^{\prime} \cap N(0, m)\right)\right),|y| \leqq Q\right\}
\end{aligned}
$$

is a bounded set in the complex plane. Since $\Phi$ is an arbitrary bounded set in $\mathscr{S}_{\alpha}$, this means that $\left\{\left(\exp (-2 \pi\langle y, t\rangle) V_{t}\right): y \in\left(C^{\prime} \backslash\left(C^{\prime} \cap N(0, m)\right)\right)\right.$, $|y| \leqq Q\}$ is a strongly bounded set in $\mathscr{S}_{\alpha}^{\prime}$. But the Fourier transform is a strongly continuous mapping of $\mathscr{S}_{\alpha}^{\prime}$ onto $\left(\mathscr{S}^{x}\right)^{\prime}$. Using this fact and (48) we conclude that $\left\{f(z): y=\operatorname{Im}(z) \in\left(C^{\prime} \backslash\left(C^{\prime} \cap N(0, m)\right)\right),|y| \leqq\right.$ $Q\}=\left\{\mathscr{F}\left[\exp (-2 \pi\langle y, t\rangle) V_{t}\right]: y=\operatorname{Im}(z) \in\left(C^{\prime} \backslash\left(C^{\prime} \cap N(0, m)\right)\right), \quad|y| \leqq\right.$ $Q\}$ is a strongly bounded set in $\left(\mathscr{S}^{\alpha}\right)^{\prime}$. This proves $(49)$, and the proof of Theorem 2 is complete.

As we have noted previously, the tube domain corresponding to the forward light cone is an example of a tubular radial domain which is 
defined by an open convex cone satisfying property $(C)$. The result of Constantinescu [4, Theorem 2] is now a special case of Theorems 1 and 2 , and the converse part of [4, Theorem 1] is a special case of Theorem 2. We note that in this special setting one does not need the notion of compact subcone, and the exponential part of (23) corresponding to $b=0$ is unnecessary. In Theorem 2 we have obtained more detailed information concerning the analytic functions than was obtained in [4, Theorem 2 and converse of Theorem 1] and have put these results in a more general setting, and Theorem 1 shows that elements of a class of analytic functions having a more general growth condition than polynomial growth obtains distributional boundary values in $\left(\mathscr{S}^{\alpha}\right)^{\prime}, \alpha=$ $\left.\alpha_{1}, \cdots, \alpha_{n}\right), \alpha_{j} \geqq 1, j=1, \cdots, n$. Results corresponding to [4, Theorem 2 and converse of Theorem 1] for functions analytic in the upper half plane and the octant may now be formulated, and they are special cases of Theorems 1 and 2.

5. Converse results. In this section we obtain converse results to Theorems 1 and 2; and throughout this section the $n$-tuple $\alpha=\left(\alpha_{1}, \cdots, \alpha_{n}\right)$ is such that $\alpha_{j} \geqq 1, j=1, \cdots, n$, as in $\S 4$.

Lemma 11 can be used to obtain a converse result corresponding to the type of $\mathscr{S}_{\alpha}^{\prime}$ distribution constructed in Theorems 1 and 2.

THeORem 3. Let $C$ be an open convex cone such that property (C) is satisfied by each compact subcone $C^{\prime} \subset C$. Let $V=D^{\gamma} g(t)$, where $g(t)$ is a continuous function on $\mathbf{R}^{n}$ which satisfies (24). Let $\operatorname{supp}(V) \subseteq C^{*}=S_{0}$. Then $V \in \mathscr{S}_{\alpha}^{\prime}$; and there exists a function $f(z) \in$ $\mathcal{U}_{C}^{0}$ such that

$$
\left\{f(z): y=\operatorname{Im}(z) \in\left(C^{\prime} \backslash\left(C^{\prime} \cap N(0, m)\right)\right),|y| \leqq Q\right\} \text { is a }
$$
strongly bounded set in $\left(\mathscr{S}^{\alpha}\right)^{\prime}$, where $Q$ is an arbitrary but fixed positive real number greater than $m$;

$$
\begin{aligned}
& f(z) \rightarrow \mathscr{F}[V] \in\left(\mathscr{T}^{x}\right)^{\prime} \text { in the weak topology of }\left(\mathscr{S}^{\alpha}\right)^{\prime} \text { as } \\
& y=\operatorname{Im} \rightarrow 0, y \in C^{\prime} \subset C \text {. }
\end{aligned}
$$

Proof. Since $C^{*}$ is a regular set (Schwartz [12, pp. 98-99]), $\operatorname{supp}(g)=\operatorname{supp}(V) \subseteq C^{*}$. Let $C^{\prime}$ be an arbitrary compact subcone of $C$ and let $m>0$ be arbitrary. By an argument as in the proof of Theorem 1, $g(t) \in \mathscr{Y}_{\alpha}^{\prime}$. Hence $V=D^{\gamma} g(t) \in \mathscr{Y}_{\alpha}^{\prime}$. We now define $f(z)$ 
as in Lemma 11. By Lemma $11, f(z) \in \mathcal{U}_{C}^{0}$. With this $f(z)$ in (55) and the fact that $V \in \mathscr{S}_{\alpha}^{\prime}$, we now proceed exactly as in the proof of Theorem 2 to obtain (56), (57), and (58).

We now wish to obtain a more general converse to Theorems 1 and 2 in that we wish to obtain a result in which one assumes that $V$ is an arbitrary element of $\mathscr{S}_{\alpha}^{\prime}$ with support in the dual cone $C^{*}$. To do so we must restrict the type of open convex cone with which we work to those which satisfy the following property, which we denote as property $\left(C^{*}\right)$ :

(C*) An open convex cone $C$ satisfies the property $\left(\mathrm{C}^{*}\right)$ if for every $\epsilon>0,\left\{t \in \mathbf{R}^{n}: 0<(-\langle y, t\rangle)<\epsilon\right\}$ is a bounded set in $\mathbf{R}^{n}$ for each fixed $y \in C$.

The cones $C=(0, \infty), C=(0, \infty)_{1} \times(0, \infty)_{2} \times \cdots \times(0, \infty)_{n}$, and the forward light cone $C=\Gamma^{+}$are examples of open convex cones which satisfy property $\left(C^{*}\right)$.

We now prove three lemmas which will be needed to obtain our general converse result.

LEMMA 12. Let $C$ be an open convex cone which satisfies property $\left(C^{*}\right)$. Let $C^{\prime}$ be an arbitrary compact subcone of $C$ and let $m>0$. Let $\lambda(t)$ be the function defined in (17) corresponding to $b=0$. Then for each fixed

$$
z \in T\left(C^{\prime}, m\right)=\mathbf{R}^{n}+i\left(C^{\prime} \backslash\left(C^{\prime} \cap N(0, m)\right)\right),(\lambda(t) \exp (2 \pi i\langle z, t\rangle)) \in \mathscr{Y}_{\alpha} .
$$

Proof. To prove the desired result we use the alternate definition of $\mathscr{S}_{\alpha}$ (Gel'fand and Shilov [9, p. 172].) Let $C^{\prime}$ be an arbitrary compact subcone of $C$ and let $m>0$ be arbitrary but fixed. It is obvious that $(\lambda(t) \exp (2 \pi i\langle z, t\rangle)) \in C^{\infty}$ as a function of $t \in \mathbf{R}^{n}$ for $z$ arbitrary but fixed in $T\left(C^{\prime}, m\right)$. We thus need to show that for any $n$-tuple $\gamma$ of nonnegative integers, there exists a constant $K_{\gamma}$ and an $n$-tuple $\left(a_{1}, \cdots, a_{n}\right)$ of positive real numbers depending on $(\lambda(t) \exp (2 \pi i\langle z, t\rangle))$ such that

$$
M^{\prime}(t)\left|D_{t}^{\gamma}\left(\lambda(t) e^{2 \pi i\langle z, t\rangle}\right)\right| \leqq K_{\gamma}, \quad t \in \mathbf{R}^{n},
$$

where

$$
M^{\prime}(t)=\exp \left(a_{1}\left|t_{1}\right|^{\left(\alpha_{1}\right)^{-1}}+\cdots+a_{n}\left|t_{n}\right|^{\left(\alpha_{n}\right)^{-1}}\right) .
$$

To obtain the estimate (59) it obviously suffices to consider $t \in \mathbf{R}^{n}$ such that $\left|t_{j}\right|>1, j=1, \cdots, n$. Further, $\lambda(t)=0$ for $t \in \mathbf{R}^{n}$ such that $\langle y, t\rangle \leqq$ $(-\epsilon), y \in\left(C^{\prime} \backslash\left(C^{\prime} \cap N(0, m)\right)\right) \subset C$, by the definition of $\lambda(t)$ in (17) corresponding to $b=0$. Thus to prove the estimate (59) it suffices to 
restrict our attention to the set $\Lambda=\left\{t \in \mathbf{R}^{n}:\left|t_{j}\right|>1, j=1, \cdots, n\right.$, and $\langle y, t\rangle>(-\epsilon)\}$, where $y=\operatorname{Im}(z) \in\left(C^{\prime} \backslash\left(C^{\prime} \cap N(0, m)\right)\right) \subset C$.

For the present we let $\left(a_{1}, \cdots, a_{n}\right)$ be any fixed $n$-tuple of positive real numbers. Let $\gamma$ be an arbitrary $n$-tuple of nonnegative integers. By the generalized Leibnitz rule

$$
M^{\prime}(t)\left|D_{t}^{\gamma}\left(\lambda(t) e^{2 \pi i(z, t)}\right)\right| \leqq M^{\prime}(t) \sum_{\beta+\rho=\gamma} \frac{\gamma !}{\beta ! \rho !}\left|D^{\beta} \lambda(t)\right|\left|z^{\rho}\right| e^{-2 \pi(y, t)}
$$

$$
\leqq M^{\prime}(t) \sum_{\beta+\rho=\gamma \mid} \frac{\gamma !}{\beta ! \rho !} K_{\beta}^{\prime}\left|z^{\rho}\right| e^{-2 \pi(y, t)}
$$

where $K_{\beta}^{\prime}$ bounds $\left|D^{\beta} \lambda(t)\right|$. Let us first restrict our attention to $t \in(\Lambda \cap\{t: 0>\langle y, t\rangle>(-\epsilon)\})$. For such $t$ we have by property $\left(C^{*}\right)$ that there exists a ball $R(0, r)$ of the origin of radius $r<\infty$ which contains $(\Lambda \cap\{t: 0>\langle y, t\rangle>(-\epsilon)\})$ (and in fact contains $\{t: 0>\langle y, t\rangle>(-\epsilon)\}$.) Thus from (61) (recall $\alpha_{j} \geq 1, j=1, \cdots, n$ )

$$
\begin{aligned}
M^{\prime}(t) & \left|D_{t}^{\gamma}\left(\lambda(t) e^{2 \pi i(z, t)}\right)\right| \\
& \leqq \exp \left(|t|\left(a_{1}+\cdots+a_{n}\right)\right) \sum_{\beta+\rho=\gamma} \frac{\gamma !}{\beta ! \rho !} K_{\beta}^{\prime}\left|z^{\rho}\right| e^{2 \pi \epsilon} \\
& \leqq \exp \left(r\left(a_{1}+\cdots+a_{n}\right)\right) \sum_{\beta+\rho=\gamma} \frac{\gamma !}{\beta ! \rho !} K_{\beta}^{\prime}\left|z^{\rho}\right| e^{2 \pi \epsilon} \\
& \leqq P
\end{aligned}
$$

for $t \in(\Lambda \cap\{t: 0>\langle y, t\rangle>(-\epsilon)\})$, where $P$ is a constant depending on the ball $R(0, r), \gamma,(\lambda(t) \exp (2 \pi i\langle z, t\rangle))$, and the $n$-tuple $\left(a_{1}, \cdots, a_{n}\right)$.

We now consider $t \in \mathbf{R}^{n}$ such that $t \in\left(\Lambda \cap C^{*} \cap R(0, r)\right)$, where $C^{*}=\left\{t \in \mathbf{R}^{n}:\langle y, t\rangle \geqq 0\right\}$ is the dual cone of $C$. For such $t, \lambda(t)=$ 1. Recalling $\alpha_{j} \geqq 1, j=1, \cdots, n$, we obtain

$$
\begin{aligned}
M^{\prime}(t) \mid D_{t}^{\gamma} & \left(\lambda(t) e^{2 \pi i(z, t)}\right)\left|=M^{\prime}(t)\right| z^{\gamma} \mid e^{-2 \pi(y, t)} \\
& \leqq \exp \left(r\left(a_{1}+\cdots+a_{n}\right)\right)\left|z^{\gamma}\right| e^{2 \pi \epsilon} \\
& \leqq P^{\prime}
\end{aligned}
$$

for $t \in\left(\Lambda \cap C^{*} \cap R(0, r)\right)$, where $P^{\prime}$ is a constant depending on the ball $R(0, r), \gamma,(\lambda(t) \exp (2 \pi i\langle z, t\rangle))$, and the $n$-tuple $\left(a_{1}, \cdots, a_{n}\right)$.

For the points $t \in \mathbf{R}^{n}$ under consideration, those $t \in \Lambda$, it remains to consider $t \in\left(\left(\Lambda \cap C^{*}\right) \backslash R(0, r)\right)$; and as in the preceding paragraph, $\lambda(t)=1$ here. For the fixed $y \in\left(C^{\prime} \backslash\left(C^{\prime} \cap N(0, m)\right)\right)$ we have by Vladimirov [14, Lemma 2, p. 223] that there exists a real number $d>0$ such that (25) holds for all $t \in C^{*}$. Thus for $t \in\left(\left(\Lambda \cap C^{*}\right) \backslash R(0, r)\right)$, 
$M^{\prime}(t)\left|D_{t}^{\gamma}\left(\lambda(t) e^{2 \pi i(z, t)}\right)\right|=M^{\prime}(t)\left|z^{\gamma}\right| e^{-2 \pi\langle y, t\rangle}$

$$
\leqq M^{\prime}(t)\left|z^{\gamma}\right| \exp (-2 \pi d|y||t|) \text {. }
$$

Since $y=\operatorname{Im}(z) \in\left(C^{\prime} \backslash\left(C^{\prime} \cap N(0 ; m)\right)\right),|y|>m>0$; and

$$
M^{\prime}(t)\left|D_{t}^{\gamma}\left(\lambda(t) e^{2 \pi i(z, t)}\right)\right|
$$

$$
\begin{aligned}
& \leqq \exp \left(a_{1}\left|t_{1}\right|+\cdots+a_{n}\left|t_{n}\right|\right)\left|z^{\gamma}\right| \exp (-2 \pi d m|t|) \\
& \leqq\left|z^{\gamma}\right| \exp \left(|t|\left(\left(a_{1}+\cdots+a_{n}\right)-2 \pi d m\right)\right)
\end{aligned}
$$

where we recall that $\alpha_{j} \geqq 1, j=1, \cdots, n$. We now choose the $n$-tuple $\left(a_{1}, \cdots, a_{n}\right)$ to consist of fixed positive real numbers $a_{j}, j=1, \cdots, n$, such that $\left(a_{1}+\cdots+a_{n}\right) \leqq 2 \pi d m$, which can be done since $d>0$ is fixed and $m>0$ is arbitrary but fixed. For this choice of $\left(a_{1}, \cdots, a_{n}\right)$ we obtain from (64)

$$
M^{\prime}(t)\left|D_{t}^{\gamma}\left(\lambda(t) e^{2 \pi i(z, t)}\right)\right| \leqq\left|z^{\gamma}\right| \leqq P^{\prime \prime},
$$

where $P^{\prime \prime}$ depends on $\gamma,(\lambda(t) \exp (2 \pi i\langle z, t\rangle))$, and the $n$-tuple $\left(a_{1}, \cdots, a_{n}\right)$. With this fixed choice of $\left(a_{1}, \cdots, a_{n}\right)$ which depends on $m$ and $d$ and hence on $C^{\prime}$ and $(\lambda(t) \exp (2 \pi i\langle z, t\rangle))$, we put

$$
K_{\gamma}^{\prime}=\max \left(P, P^{\prime}, P^{\prime \prime}\right) \text {. }
$$

Combining (62), (63), and (65), we thus have that

$$
M^{\prime}(t)\left|D_{t}^{\gamma}\left(\lambda(t) e^{2 \pi i\langle z, t}\right)\right| \leqq K_{\gamma}^{\prime}, \quad t \in \Lambda,
$$

where $K_{\gamma}^{\prime}$ is a constant depending on $\gamma,(\lambda(t) \exp (2 \pi i\langle z, t\rangle))$, and the $n$-tuple $\left(a_{1}, \cdots, a_{n}\right)$ (and possibly on the ball $R(0, r)$.) (59) is now easily obtained from (66) and the fact that $\lambda(t)=0$ for $t \in \mathbf{R}^{n}$ such that $\langle y, t\rangle \leqq(-\epsilon)$. The proof is complete.

LEMMA 13. Let $C$ be an open convex cone which satisfies property $\left(C^{*}\right)$. Let $V \in \mathscr{S}_{\alpha}^{\prime}$ with $\operatorname{supp}(V) \subseteq C^{*}=\left\{t: u_{C}(t) \leqq 0\right\}$. Then $f(z)=$ $\langle V, \exp (2 \pi i\langle z, t\rangle)\rangle \in \mathcal{U}_{c}^{0}$.

Proof. Let $C^{\prime}$ be an arbitrary compact subcone of $C$, and let $m>0$. Let $z$ be an arbitrary but fixed point of $T\left(C^{\prime}, m\right)=$ $\mathbf{R}^{n}+i\left(C^{\prime} \backslash\left(C^{\prime} \cap N(0, m)\right)\right)$. By Lemma 12, $\quad(\lambda(t) \exp (2 \pi i\langle z, t\rangle)) \in$ $\mathscr{S}_{\alpha}$. Since $V \in \mathscr{S}_{\alpha}^{\prime}$ and $\operatorname{supp}(V) \subseteq C^{*}$, we have 


$$
f(z)=\left\langle V, e^{2 \pi i\langle z, t\rangle}\right\rangle=\left\langle V, \lambda(t) e^{2 \pi i\langle z, t\rangle}\right\rangle, \quad z \in T\left(C^{\prime}, m\right),
$$

is well defined as a function of $z \in T\left(C^{\prime}, m\right)$. By [9, Chapter IV, §4], $(\lambda(t) \exp (2 \pi i\langle z, t\rangle)) \in \mathscr{S}_{\alpha}$ implies $\left(\left(t_{j}\right) \quad \lambda(t) \exp (2 \pi i\langle z, t\rangle)\right) \in \mathscr{S}_{\alpha}, \quad j=$ $1, \cdots, n$. Using the linearity and continuity of $V \in \mathscr{S}_{\alpha}^{\prime}$, it is straightforward to show that for each $j=1, \cdots, n$

$$
\begin{aligned}
\frac{\partial f(z)}{\partial z_{j}} & =\lim _{\Delta z_{\jmath} \rightarrow 0}\left(\left(f\left(z_{1}, \cdots, z_{\jmath-1}, z_{\jmath}+\Delta z_{l}, z_{\jmath+1}, \cdots, z_{n}\right)-f(z)\right) / \Delta z_{j}\right) \\
& \left\langle V, \frac{\partial}{\partial z_{\jmath}}\left(\lambda(t) e^{2 \pi i\langle z . t)}\right)\right\rangle \\
& =2 \pi i\left\langle V,\left(t_{\jmath}\right) \lambda(t) e^{2 \pi \imath\langle z, t\rangle}\right\rangle .
\end{aligned}
$$

Thus for $z_{1}, z_{2}, \cdots, z_{j-1}, z_{j+1}, \cdots, z_{n}$ fixed, $f(z)$ is analytic as a function of $z_{\text {J }}$ in the projection of $T\left(C^{\prime}, m\right)$ into the $j$ th coordinate plane, $j=$ $1, \cdots, n$. By Hartog's theorem [1, Chapter VII] $f(z)$ is analytic in $T\left(C^{\prime}, m\right)$.

It remains to show that $f(z)$ satisfies the boundedness condition (23) for $b=0$. Since $(\lambda(t) \exp (2 \pi i\langle z, t\rangle)) \in \mathscr{S}_{\alpha}$, then there exists an $n$ - tuple $A=\left(A_{1}, \cdots, A_{n}\right)$ such that $(\lambda(t) \exp (2 \pi i\langle z, t\rangle)) \in \mathscr{S}_{\alpha \cdot A}$. In fact this $n$-tuple $A$ can be obtained from the $n$-tuple $\left(a_{1}, \cdots, n\right)$ chosen in the proof of Lemma 12 by the relation stated in (1). Also $\mathscr{S}_{\alpha}^{\prime} \subset \mathscr{S}_{\alpha . A}^{\prime}$ and $V \in \mathscr{S}_{\alpha}^{\prime}$ imply that $V \in \mathscr{S}_{\alpha, A}^{\prime}$. Now $\mathscr{S}_{r, A}$ is a complete countably normed locally convex topological vector space whose topology is defined by the sequence of norms (3), and $V$ is a continuous linear functional on $\mathscr{S}_{\alpha, A}$. Thus there exists a positive constant $M$ and a value of $p=2,3,4, \cdots$ such that

$$
|f(z)| \leqq M\left\|\lambda(t) e^{2 \pi \iota(z, t)}\right\|_{p} .
$$

We may now proceed exactly as in the proof of Lemma 12 with $M_{p}(t)$ defined in (2) replacing the function $M^{\prime}(t)$ defined in (60) to obtain inequalities similar to (62), (63), and (65) and conclude for each $\gamma$, $|\gamma| \leqq p$, that

$$
M_{p}(t)\left|D_{t}^{\gamma}\left(\lambda(t) e^{2 \pi i(z, t)}\right)\right| \leqq K_{\gamma}(1+|z|)^{N_{\gamma}}, \quad t \in \mathbf{R}^{n},
$$

where $z \in T\left(C^{\prime}, m\right), K_{\gamma}$ is a constant depending on $\gamma$ and on $C^{\prime}$, and $N_{\gamma}$ is a positive real number depending on $\gamma$. (68) holds independently of $t \in \mathbf{R}^{n}$ and for each $\gamma,|\gamma| \leqq p$. Thus from (67) and (68) we obtain the existence of a constant $K^{\prime}$ depending on $\gamma$ and $C^{\prime}$ and a constant $N$ such that 


$$
|f(z)| \leqq M K^{\prime}(1+|z|)^{N}, \quad z \in T\left(C^{\prime}, m\right) .
$$

Since $\exp (2 \pi \sigma|y|)>1$ for all $\sigma>0, y \in\left(C^{\prime} \backslash\left(C^{\prime} \cap N(0, m)\right)\right)$, we see that $f(z)$ satisfies (23) for $b=0$; and the proof is complete.

Lemma 14. Let the hypotheses of Lemma 13 be satisfied, and let $\psi \in \mathscr{S}^{\alpha}$. Then

$$
\left\langle\left\langle V, \lambda(t) e^{2 \pi i\langle z, t\rangle}\right\rangle, \psi(x)\right\rangle=\left\langle V, \lambda(t) e^{-2 \pi\langle y, t\rangle} \check{\phi}(t)\right\rangle,
$$

$\mathrm{z}=\mathrm{x}+\mathrm{iy} \in \mathrm{T}\left(\mathrm{C}^{\prime}, \mathrm{m}\right)$, where $\mathrm{C}^{\prime}$ is an arbitrary compact subcone of $\mathrm{C}$, $\mathrm{m}>0$, and $\check{\phi}(\mathrm{t})=\phi(-\mathrm{t}) \in \mathscr{S}_{\alpha}$ such that

$$
\check{\phi}(\mathrm{t})=\mathscr{F}^{-1}[\psi(\mathrm{x}) ;(-\mathrm{t})]=\int_{\mathbf{R}^{n}} \psi(x) e^{2 \pi \iota\langle x, t)} d x .
$$

Proof. By Lemma 13, $f(z)=\langle V, \lambda(t) \exp (2 \pi i\langle z, t\rangle)\rangle \in \mathcal{U}_{c}^{0}$. We now let $y=\operatorname{Im}(z) \in\left(C^{\prime} \backslash\left(C^{\prime} \cap N(0, m)\right)\right)$ be fixed. Because of the growth behavior (23) for $b=0$ and the fact that $f(z)$ is continuous as a function of $x=\operatorname{Re}(z), f(z) \in\left(\mathscr{S}^{\alpha}\right)^{\prime}$ as a function of $x=\operatorname{Re}(z)$. In fact the product of $f(z)$ with an element of $\mathscr{S}^{\alpha}$ is Riemann integrable with respect to $x=\operatorname{Re}(z)$; and the Reimann integral can be approximated by Riemann sums. Consider the Cartesian product on $n$ intervals [$Y, Y] \times \cdots \times[-Y, Y]$. Divide the coordinate axes in $\mathbf{R}^{n}$ into partitions, and let $\Delta v_{j}$ be the volume and $\left(x_{1}, \cdots, x_{n_{j}}\right)$ be a point of the $j$ th small parallelpiped. By the continuity and linearity of $V \in \mathscr{S}_{\alpha}^{\prime}$ we obtain

$$
\langle f(z), \psi(\dot{x})\rangle=\lim _{Y \rightarrow \infty} \int_{-Y}^{Y} \cdots \int_{-Y}^{Y}\left\langle V, \lambda(t) e^{2 \pi \iota\langle z, t\rangle}\right\rangle \psi(x) d x
$$

$$
\begin{gathered}
=\left\langle V, \lim _{Y \rightarrow \infty} \lim _{J \rightarrow \infty} \sum_{j=1}^{J^{n}} \lambda(t) \exp \left(2 \pi i\left\langle\left(x_{1_{j}}+i y_{1}, \cdots, x_{n_{j}}+i y_{n}\right), t\right\rangle\right)\right. \\
\left.\psi\left(x_{1_{j}}, \cdots, x_{n_{1}}\right) \Delta v_{j}\right\rangle,
\end{gathered}
$$

where $\psi \in \mathscr{S}^{\alpha}$. It is straightforward to show that the right hand side of the last equality in (71) converse in $\mathscr{S}_{\alpha}$ to

$$
\lambda(t) \int_{\mathbf{R}^{n}} \psi(x) e^{2 \pi i(z, t\rangle} d x=\lambda(t) e^{-2 \pi\langle y, t\rangle} \check{\phi}(t),
$$

where $\check{\phi}(t)=\phi(-t) \in \mathscr{S}_{x}$ such that (70) holds. Thus from (71) and the fact that $V \in \mathscr{S}_{\alpha}^{\prime}$ we have (69).

We now obtain the desired converse to Theorems 1 and 2. 
THEOREM 4. Let $C$ be an open convex cone which satisfies property $\left(C^{*}\right)$. Let $C^{\prime}$ be an arbitrary compact subcone of $C$ and let $m>0$. Let $V \in \mathscr{S}_{\alpha}^{\prime}$ with $\operatorname{supp}(V) \subseteq C^{*}=\left\{t: u_{C}(t) \leqq 0\right\}$. Then there exists a function $f(z) \in \mathcal{U}_{C}^{0}$ such that

(73) $f(z)=\mathscr{F}\left[e^{-2 \pi(y, t)} V_{t}\right], z=x+i y \in T\left(C^{\prime}, m\right)$, where the equality (73) is in $\left(\mathscr{S}^{\alpha}\right)^{\prime}$;

$$
\left\{f(z): y=\operatorname{Im}(z) \in\left(C^{\prime} \backslash\left(C^{\prime} \cap N(0, m)\right)\right),|y| \leqq Q\right\} \text { is a }
$$
strongly bounded set in $\left(\mathscr{S}^{\alpha}\right)^{\prime}$, where $Q$ is an arbitrary but fixed positive real number greater than $m$;

$$
\begin{aligned}
& f(z) \rightarrow \mathscr{F}[V] \in\left(\mathscr{S}^{x}\right)^{\prime} \text { in the weak topology of }\left(\mathscr{S}^{\alpha}\right)^{\prime} \text { as } \\
& y=\operatorname{Im}(z) \rightarrow 0, y \in C^{\prime} \cdot \subset C .
\end{aligned}
$$

Proof. By Lemma 13, the function $f(z)$ defined in (72) exists under the convention

$$
f(z)=\left\langle V, e^{2 \pi i\langle z, t)}\right\rangle=\left\langle V, \lambda(t) e^{2 \pi i\langle z, t\rangle}\right\rangle, \quad z \in T\left(C^{\prime}, m\right),
$$

where supp $(V) \subseteq C^{*}$ and $\lambda(t)$ is defined in (17) corresponding to $b=0$; and $f(z)$ is an element of $U_{C}^{0}$. As in Lemma 14, there exists an element $\check{\phi}(t)=\phi(-t) \in \mathscr{S}_{x}$ such that $\psi(x)=\mathscr{F}[\check{\phi}(t) ; x]$ and $(70)$ holds (recall the proof of Theorem 1.) For the moment we let $y=\operatorname{Im}(z)$ be an arbitrary but fixed point of $\left(C^{\prime} \backslash\left(C^{\prime} \cap N(0, m)\right)\right)$. From Lemma 14, (4), and the fact that $\psi(x)=\mathscr{F}[\check{\phi}(t) ; x]$ we obtain

$$
\langle f(z), \psi(x)\rangle=\left\langle V, \lambda(t) e^{-2 \pi\langle y, t)} \check{\phi}(t)\right\rangle=\left\langle\mathscr{F}\left[e^{-2 \pi(y, t)} V_{t}\right], \psi(x)\right\rangle,
$$

where $\operatorname{supp}(V) \subseteq C^{*}$ implies $(\lambda(t) V)=V$ in $\mathscr{S}_{\alpha}^{\prime}$; and (73) is proved. Using the representation (73) we may now prove (74) exactly as we proved (49) in Theorem 2.

It remains to prove (75). From Lemma $4,(\lambda(t) \exp (-2 \pi\langle y, t\rangle)$ $\check{\phi}(t)) \rightarrow(\lambda(t) \check{\phi}(t))$ in $\mathscr{S}_{x}$ as $y \rightarrow 0, y \in C^{\prime} \subset C$. Since $V \in \mathscr{S}_{\alpha}^{\prime}$ and $\operatorname{supp}(V) \subseteq C^{*}$, we have

$$
\left\langle V, \lambda(t) e^{-2 \pi\langle y, t\rangle} \check{\phi}(t)\right\rangle \rightarrow\langle V, \lambda(t) \check{\phi}(t)\rangle=\langle V, \check{\phi}(t)\rangle
$$

as $y \rightarrow 0, y \in C^{\prime} \subset C$. From (69), (76), and (4) we have (recall $m>0$ is arbitrary)

$$
\langle f(z), \psi(x)\rangle \rightarrow\langle V, \check{\phi}(t)\rangle=\langle\mathscr{F}[V], \psi(x)\rangle
$$


as $\quad y \rightarrow 0, \quad y \in C^{\prime} \subset C, \quad$ where $\quad \psi(x)=\mathscr{F}[\check{\phi}(t) ; x] ; \quad$ and $\mathscr{F}[V] \in\left(\mathscr{S}^{x}\right)^{\prime}$. This proves $(75)$, and the proof is complete.

From Lemma 9 and the hypothesis on $V$ in Theorems 3 and 4, we see that we may also conclude in these theorems that (exp ($\left.2 \pi\langle y, t\rangle) V_{t}\right) \in \mathscr{S}_{\alpha}^{\prime}$ for all $y \in C$. Thus for $C$ being the forward light cone in Theorem 4, the hypothesis of Theorem 4 implies the hypothesis and conclusion of the sufficiency of [4, Theorem 1]; and Theorem 4 is a generalization of the sufficiency of $[4$, Theorem 1].

6. Conclusions and applications. We have extended (Theorems 2, 3, 4) the results of Constantinescu [4, Theorems 1 and 2] to the setting of functions analytic in tubular radial domains and for distributions in $\left(\mathscr{S}^{x}\right)^{\prime}, \alpha=\left(\alpha_{1}, \cdots, \alpha_{n}\right), \alpha_{j} \geqq 1, j=1, \cdots, n$. In our theorems we have also obtained new representations and information concerning the analytic functions which have $\left(\mathscr{S}^{\alpha}\right)^{\prime}$ boundary values and which are representable as the Fourier-Laplace transform of the inverse Fourier transform of the boundary value. Further, we have shown (Theorem 1) that functions having a more general growth condition than those considered by Constantinescu have distributional boundary values in the weak topology of $\left(\mathscr{S}^{\alpha}\right)^{\prime}$. The main theorems in this paper have been established with the aid of several new lemmas, which we have proved here, concerning the spaces of type $\mathscr{S}$ and their dual spaces. The distributional boundary value results have as application and as special cases not only the results of Constantinescu for functions analytic in tube domains corresponding to the light cone but also for the corresponding functions analytic in half planes in $\mathbf{C}^{1}$ and octants in $\mathbf{C}^{n}$.

Aside from the mathematical interest of the results presented in this paper concerning distributional boundary values of analytic functions in $\mathbf{C}^{n}$ and the spaces of type $\mathscr{S}$ and their dual spaces, the analysis developed here and in the paper [2] of one of the authors is of interest in applications in the sense of the last sentence in the previous paragraph. In applications to quantum field theory, distributional boundary value results are of interest for functions analytic in tube domains corresponding to the light cone and in half planes. The techniques which we have established here and in [2] in our general setting for the construction of the inverse Fourier transform of the boundary value from the analytic function and the establishment of the support set (see Theorem 1) can be applied to the special domains and can be used to establish similar results for other types of analytic functions which are of interest in quantum field theory. Further, our technique in establishing the boundary value result is similarly applicable (recall Theorems 1 and 2). The techniques employed in the proof of analyticity and growth in Lemma 13 and in the proof of "change of 
order of integration" in Lemma 14 are useful in the setting for applications especially if there is no representation theorem for the distributions involved.

We note, for example, the recent paper of Constantinescu and Thalheimer [5] in which Euclidean Green's functions for Jaffe fields are studied. Theorems 1 and 2 of [5] involve the study of the FourierLaplace transform of "ultradistributions" and are used to obtain the field theory results. These theorems are obtained in the terminology of the present paper restricted to 1-dimension. That is, functions analytic in $\mathbf{R}^{1}+i C, C$ being an open convex cone in $\mathbf{R}^{\prime}$, and satisfying a growth condition for imaginary part in compact subcones of $C$ are shown to be representable as a Fourier-Laplace transform of a certain generalized function having support in the dual cone of $C$ in $\mathbf{R}^{1}$ and are shown to have a distributional boundary value. Also a representation as in (48) of this paper is used. The techniques involved in the proof of the support set, for example, are essentially the same that we give in the present paper and in [2] restricted to 1-dimension. Similarly, other techniques involved in these two theorems of Constantinescu and Thalheimer are essentially 1-dimensional restrictions of our techniques. (It is interesting to note that the test spaces of functions and the corresponding spaces of generalized functions studied in [5] are closely related to the spaces of type $\mathscr{S}$ and their dual spaces [5, pp. 3-4].) The point is that even for other distribution spaces and for the mathematics of fie'd theories other than the one introduced by Constantinescu [4], with which the results of this paper are concerned, techniques employed in this paper for the general setting of functions analytic in tubular radial domains can be adapted to prove corresponding results for other field theories and for functions analytic in special cases of tubular radial domains. Our techniques in the general setting indicate techniques to be used in the settings of application to quantum field theory. (We could have chosen any number of other papers on quantum field theory as examples of the point we make above. We have chosen [5] because of its use of cone terminology and the relation of the test functions employed in [5] to the spaces of type $\mathscr{S}$.)

We shall now consider some mathematical applications of the results which we have obtained in this paper.

A well known result of Bochner [1, p. 92] states that every "tube" $[1$, p. 90] has a uniquely determined largest analytic completion which is the convex envelope of the given "tube." Stated in another form, this result says that if $f(z)$ is analytic in the tube $T^{B}=\mathbf{R}^{n}+i B$, where $B \subset \mathbf{R}^{n}$ is a domain, then $f(z)$ has an analytic extension to the convex envelope $O\left(T^{B}\right)=T^{O(B)}=\mathbf{R}^{n}+i O(B)$ of $T^{B}$. We now desire to prove a result which is similar to this theorem of Bochner but more general in the sense that the given function is analytic in a subset of $C^{n}$ of the form $T^{C}$ 
where $C$ is an open cone which does not have to be connected. (Recall that in Bochner's result the imaginary (real, respectively,) part of the space of analyticity must be a domain, hence must be connected.) Using the distributional boundary value results presented in this paper, we show that certain functions which are analytic in $T^{C}$, where $C$ is an open cone which is the finite union of open convex cones, have an analytic extension to $T^{O(C)}$, the convex envelope of $T^{C}$. (Recall from $\$ 2$ that $O(C)$ denotes the convex envelope of the cone $C$.) Further, we explicitly construct the extension function.

Let $C$ be an open cone such that $C=\cup_{j=1}^{r} C_{j}, r<\infty$, where the $C_{j}$ are open convex cones such that property (C) is satisfied by each compact subcone $C_{j}^{\prime} \subset C_{j}, j=1, \cdots, r$. Let $C^{\prime}$ be an arbitrary compact subcone of $C$. Let $f(z)$ be analytic in $T\left(C^{\prime}, m\right), m>0$ being arbitrary, and satisfy (23) for $b=0$. Let us now restrict $z$ to $T^{C_{\mathrm{i}}}=\mathbf{R}^{n}+i C_{\mathrm{j}}$, $j=1, \cdots, r$. Then $f(z) \in \mathcal{U}_{C_{\mathrm{s}}}^{0}, j=1, \cdots, r$. By Theorem 2 there exist unique elements $V_{j} \in \mathscr{S}_{\alpha}^{\prime}$ with $\sup P\left(V_{j}\right) \subseteq C_{j}^{*}$ such that

$$
f(z) \rightarrow \mathscr{F}\left[V_{j}\right]=U_{1} \in\left(\mathscr{S}^{x}\right)^{\prime}
$$

in the weak topology of $\left(\mathscr{S}^{x}\right),^{\prime}$ as $y=\operatorname{Im}(z) \rightarrow 0, y \in C_{j}^{\prime} \subset C_{j}, j=$ $1, \cdots, r$. Using this fact, we now obtain conditions under which functions $f(z)$ that are analytic in $T^{C}$ have an analytic extension to $T^{O(C)}$.

THEOREM 5. Let $C$ be an open cone such that $O(C)$ satisfies property $\left(\mathrm{C}^{*}\right)$ and $C=\cup_{j=1}^{r} C_{j} r<\infty$, where each $C_{j}$ is an open convex cone such that property (C) is satisfied by each compact subcone $C_{,}^{\prime} \subset C_{j}, j=1, \cdots, r$. Let $f(z)$ be analytic in $T\left(C^{\prime}, m\right), m>0$, and satisfy (23) for $b=0$. Let the $\left(\mathscr{S}^{\alpha}\right)^{\prime}$ boundary values $U_{j}$ of $f(z)$ which exist from each connected component $T^{C_{1}}, j=1, \cdots, r$, be equal. There exists an element $V \in \mathscr{S}_{\alpha}^{\prime}$ with $\operatorname{supp}(V) \subseteq\left\{t: u_{O(C)}(t) \leqq 0\right\}$ such that

$$
\begin{gathered}
\left(\exp (-2 \pi\langle y, t\rangle) V_{t}\right) \in \mathscr{S}_{\alpha}^{\prime} \text { for all } y \in C ; \\
f(z)=\left\langle V, e^{2 \pi i\langle z, t\rangle}\right\rangle, \quad z \in T\left(C^{\prime}, m\right), C^{\prime} \subset C ; \\
f(z)=\mathscr{F}\left[e^{-2 \pi\langle y, t\rangle} V_{t}\right], z=x+i y \in T\left(C^{\prime}, m\right), \quad C^{\prime} \subset C, \\
\text { where the equality }(79) \text { is in }\left(\mathscr{S}^{x}\right)^{\prime} ; \\
\left\{f(z): y=\operatorname{Im}(z) \in\left(C^{\prime} \backslash\left(C^{\prime} \cap N(0, m)\right)\right),|y| \leqq Q\right\} \text { is } \\
\text { a strongly bounded set in }\left(\mathscr{S}^{\alpha}\right)^{\prime}, \text { where } Q \text { is an } \\
\text { arbitrary but fixed positive real number greater than } \\
m ;
\end{gathered}
$$

and there exists an element $F(z) \in \mathcal{U}_{\alpha(C)}^{0}$ which is the analytic extension of $f(z)$. 
Proof. As in the paragraph preceding the statement of this theorem, we apply Theorem 2 to $f(z)$ restricted to each of $T^{c_{1}}$, $j=1, \cdots, r$, and obtain elements $V_{j} \in \mathscr{Y}_{\alpha}^{\prime}$ with $\operatorname{supp}\left(V_{j}\right) \subseteq C_{j}^{*}$ such that $f(z) \rightarrow \mathscr{F}\left[V_{j}\right]=U_{j} \in\left(\mathscr{S}^{x}\right)^{\prime} \quad$ as $\quad y=\operatorname{Im}(z) \rightarrow 0, \quad y \in C_{j}^{\prime} \subset C_{j}$. By hypothesis $U_{1}=U_{2}=\cdots=U_{r}$ in $\left(\mathscr{S}^{x}\right)^{\prime}$, and we call this common value $U$. But $U_{j}=\mathscr{F}\left[V_{j}\right]$ implies $V_{j}=\mathscr{F}^{-1}\left[U_{j}\right], j=1, \cdots, r$. Since the inverse Fourier transform is a one-to-one mapping of $\left(\mathscr{S}^{x}\right)^{\prime}$ onto $\mathscr{S}_{\alpha}^{\prime}$, we have $V_{1}=V_{2}=\cdots=V_{r}$ in $\mathscr{S}_{\alpha}^{\prime}$. We call this common value $V$, and $V \in \mathscr{S}_{\alpha}^{\prime}$. Since $\operatorname{supp}\left(V_{J}\right) \subseteq\left\{t: u_{C_{1}}(t) \leqq 0\right\}, j=1, \cdots, r$, then $V$ vanishes on $\cup_{j=1}^{r}\left\{t: u_{C_{j}}(t)>0\right\}$. Now

$$
u_{C}(t)=\max _{j=1, \cdots, r}\left(u_{C}(t)\right),
$$

and from the definition of $\rho_{C}$ in $\S 2$, we have $u_{O(C)}(t) \leqq\left(\rho_{C} u_{C}(t)\right)$. Thus

$$
u_{O(C)}(t) \leqq\left(\rho_{C}\right) \max _{j=1, \cdots, r}\left(u_{C_{j}}(t)\right),
$$

and by a lemma of Vladimirov [14, Lemma 3, p. 220], $1 \leqq \rho_{C}<$ $+\infty$. Now if $t \in\left\{t: u_{O(C)}(t)>0\right\}$, then by (81) $t \in$ $\left\{t: \max _{j=1, \ldots, r}\left(u_{\mathrm{C}_{\mathrm{j}}}(t)\right)>0\right\}$. Hence $t \in \cup_{j=1}^{r}\left\{t: u_{\mathrm{C}_{\mathrm{j}}}(t)>0\right\}$, and on this set $V$ vanishes. Thus $V$ vanishes if $t \in\left\{t: u_{O(C)}(t)>0\right\}$ which implies that $\operatorname{supp}(V) \subseteq\left\{t: u_{O(C)}(t) \leqq 0\right\}$.

Applying (46), (47), and (48) of Theorem 2 to $f(z)$ restricted to each $T^{c_{i}}$, we have

$$
\begin{aligned}
& \left(\exp (-2 \pi\langle y, t\rangle) V_{j}\right) \in \mathscr{S}_{\alpha}^{\prime}, y \in C_{j}, \\
& f(z)=\left\langle V_{j}, e^{2 \pi i\langle z, t\rangle}\right\rangle, \\
& f(z)=\mathscr{F}\left[e^{-2 \pi\langle y, t\rangle} V_{j}\right],
\end{aligned}
$$

with the latter equality being in $\left(\mathscr{S}^{x}\right)^{\prime}$; and these hold for $z \in T\left(C_{j}^{\prime}, m\right)$, $C_{j}^{\prime} \subset C_{j}, j=1, \cdots, r$. Since $V=V_{1}=V_{2}=\cdots=V_{r}$, then (77), (78), and (79) are obtained. Further, (80) follows from (49) in a similar fashion.

We now consider

$$
F(z)=\left\langle V, e^{2 \pi i\langle z, t)}\right\rangle, \quad z \in T^{O(C)} .
$$

Since $V \in \mathscr{Y}_{\alpha}^{\prime}$ and $\operatorname{supp}(V) \subseteq\left\{t: u_{O(C)}(t) \leqq 0\right\}$, we have by Theorem 4 that $F(z) \in \mathscr{U}_{\alpha C C}^{0}$. Because of $(78)$ we see further that $F(z)$ is the desired analytic extension of $f(z)$ to $T^{O(C)}$, and the proof is complete.

Note that (78) provides a representation of $f(z)$ for $z$ in the space of analyticity of $f(z)$. In addition to its relation to the analytic extension 
theorem of Bochner, Theorem 5 is also a generalization of Theorem 2 to tubes over disconnected cones. It is evident that Theorem 5 is not a precise extension of Bochner's result to tubes over disconnected cones for the functions and cone considered since in the first place the given function $f(z)$ is not assumed to be analytic in the whole of $T^{c}$ but only in sets of the form $T\left(C^{\prime}, m\right), C^{\prime} \subset C, m>0$ arbitrary; and in the second place, the extension function is not analytic in the whole of $T^{O(C)}$ but in similar type sets in $T^{o(C)}$. However, Theorem 5 is an extension of Bochner's result to tubes over disconnected cones for the functions considered in the sense that our constructed extension function equals the given function on the sets of analyticity of the given function and is itself analytic on precisely the same type sets in the whole of $T^{O(C)}$.

Theorem 5 has as a corollary the following result.

Corollary 1. Let $C=((-\infty, 0) \cup(0, \infty))$. Let $f(z)$ be analytic in $|\operatorname{Im}(z)|>m$ for any $m>0$ and satisfy (23) there for $b=0$. Let $U_{1}=U_{2}$ where $U_{1}\left(U_{2}\right)$ is the weak $\left(\mathscr{S}^{\alpha}\right)^{\prime}$ boundary value of $f(z)$ from the upper (lower) half plane. There exists an element $V \in \mathscr{Y}_{\alpha}^{\prime}$ with $\operatorname{supp}(V)=\{0\}$ such that

$$
\begin{aligned}
& \left(\exp (-2 \pi\langle y, t\rangle) V_{t}\right) \in \mathscr{S}_{\alpha}^{\prime} \text { for all } y \in C ; \\
& f(z)=\left\langle V, e^{2 \pi i(z, t)}\right\rangle,|\operatorname{Im}(z)|>m>0 ; \\
& f(z)=\mathscr{F}\left[e^{-2 \pi(y, t)} V\right],|\operatorname{Im}(z)|>m>0, \text { in }\left(\mathscr{S}^{\alpha}\right)^{\prime} ; \\
& \{f(z):|\operatorname{Im}(z)|>m>0,|\operatorname{Im}(z)| \leqq Q, m<Q\} \text { is a strongly }
\end{aligned}
$$

bounded set in $\left(\mathscr{S}^{\alpha}\right)^{\prime}$.

The proof is obtained by observing that $O(C)=\mathbf{R}^{1}$ and $\left\{t: u_{O(C)}(t) \leqq 0\right\}=\{0\}$ and applying the conclusions of Theorem 5. In one dimension we do not need the notion of compact subcones. Note that since $(O(C) \backslash C)=\{0\}, f(z)$ is its own extension as the representation $f(z)=\langle V, \exp (2 \pi i\langle z, t\rangle)\rangle,|\operatorname{Im}(z)|>m>0$, indicates.

A well known problem in classical analysis, which is sometimes referred to as the Hilbert boundary value problem, is as follows: given a function $G(\xi)$ on the boundary of a domain $D$ in $\mathbf{C}^{\prime}$, find analytic functions in $D$ and in the complement of $\bar{D}$ such that the sum of the analytic functions has $G(\xi)$ as boundary value. In particular this problem has considerable interest when the boundary is the real axis and the domains of analyticity are the upper and lower half planes. We now formulate this problem in $\mathbf{C}^{n}$ with respect to the distributions in $\left(\mathscr{S}^{\alpha}\right)^{\prime}, \alpha=\left(\alpha_{1}, \cdots, \alpha_{n}\right), \alpha_{j} \geqq 1, j=1, \cdots, n$. In particular we shall show, using our distributional boundary value results, that any element $U \in\left(\mathscr{G}^{\alpha}\right)^{\prime}$ can be represented as the boundary value of a sum of 
analytic functions on the distinguished boundary of a tubular cone which is the union of tubular radial domains; that is, we show that any element $U \in\left(\mathscr{S}^{\alpha}\right)^{\prime}$ can be decomposed as a sum of elements in $\left(\mathscr{S}^{\alpha}\right)^{\prime}$ each of which is the boundary value of an analytic function in a tubular radial domain.

Let $C$ be an open cone such that $C=\cup_{j=1}^{r} C_{j}, r<\infty$, where each of the $C_{j}$ are open convex cones which satisfy property $\left(\mathrm{C}^{*}\right)$ and where the dual cones $C_{i}^{*}, j=1, \cdots, r$, satisfy

$$
\mathbf{R}^{n} \backslash \bigcup_{j=1}^{r} C_{j}^{*} \quad \text { and } \quad C_{i}^{*} \cap C_{k}^{*}, j \neq k, \begin{aligned}
& j=1, \cdots, r \\
& k=1, \cdots, r
\end{aligned}
$$

are sets of Lebesgue measure zero. Examples of open cones $C$ which satisfy the above properties are $C=((-\infty, 0) \cup(0, \infty))$ in one dimension and $C=\cup_{\delta}\left\{y \in \mathbf{R}^{n}: \delta_{j} y_{j}>0, \delta=\left(\delta_{1}, \cdots, \delta_{n}\right), \delta_{j}= \pm 1, j=1, \cdots, n\right\}$, where $r=2^{n}$.

Theorem 6. Let $C=\cup_{\mathrm{j}=1}^{r} C_{\mathrm{j}}, r<\infty$, be an open cone such that the properties for the $C_{j}$ and $C_{j}^{*}, j=1, \cdots, r$, in the preceding paragraph are satisfied. Let $U \in\left(\mathscr{S}^{\alpha}\right)^{\prime}$. Then $U=\sum_{j=1}^{r} U_{j}$, where $U_{j} \in\left(\mathscr{S}^{\alpha}\right)^{\prime}, j=$ $1, \cdots, r$; each $U_{1}$ is the weak $\left(\mathscr{S}^{\alpha}\right)^{\prime}$ boundary value of a function $f_{j}(z) \in \mathcal{U}_{C_{j}}^{0} ;$ and each $U_{j}=\mathscr{F}\left[V_{j}\right]$ where $V_{j} \in \mathscr{S}_{\alpha}^{\prime}$ and $\operatorname{supp}\left(V_{j}\right) \subseteq C_{i}^{*}$, $j=1, \cdots, r$.

Proof. There exists $V \in \mathscr{S}_{\alpha}^{\prime}$ such that $V=\mathscr{F}^{-1}[U]$ and $U=$ $\mathscr{F}[V]$. Put

$$
\left(V_{j}\right)_{t}=\left\{\begin{array}{c}
V_{t}, t \in C_{j}^{*}, \\
0, t \in\left(\mathbf{R}^{n} \backslash C_{j}^{*}\right),
\end{array}\right.
$$

$j=1, \cdots, r$, where $V_{t}$ and $\left(V_{j}\right)_{t}$ indicate that the distributions $V$ and $V_{j}$ are operating on the variable $t$. We have $V_{j} \in \mathscr{S}_{\alpha}^{\prime}$ and $\operatorname{supp}\left(V_{j}\right) \subseteq C_{j}^{*}$, $j=1, \cdots, r$. We now put $f_{j}(z)=\left\langle V_{j}, \quad \exp (2 \pi i\langle z, t\rangle)\right\rangle, \quad z \in T^{C_{j}}, j=$ $1, \cdots, r$. By Theorem $4, f_{j}(z) \in \mathcal{U}_{C_{j}}^{0}$ and $f_{j}(z) \rightarrow \mathscr{F}\left[V_{j}\right]=U_{j} \in\left(\mathscr{S}^{x}\right)^{\prime}$ in the weak topology of $\left(\mathscr{S}^{x}\right)^{\prime}$ as $y=\operatorname{Im}(z) \rightarrow 0, \quad y \in C_{j}^{\prime} \subset C_{j}, j=$ $1, \cdots, r$. By the hypothesis (82) on the dual cones $C_{i}^{*}, j=1, \cdots, r$, we have $V=\sum_{j=1}^{r} V_{j}$ in $\mathscr{S}_{\alpha}^{\prime}$. Thus (4) and linearity yield

$$
\langle U, \psi\rangle=\langle V, \phi\rangle=\sum_{j=1}^{r}\left\langle V_{j}, \phi\right\rangle=\sum_{j=1}^{r}\left\langle U_{j}, \psi\right\rangle=\left\langle\sum_{j=1}^{r} U_{j}, \psi\right\rangle,
$$

$\phi \in \mathscr{S}_{x}, \psi=\hat{\phi} \in \mathscr{S}^{\alpha}$, and the proof is complete.

As noted in the introduction to the Hilbert boundary value problem considered in Theorem 6 , the complex plane $\mathbf{C}^{1}$ is the setting in which 
this problem has most interest classically, In the following result we obtain the restriction of Theorem 6 to this classical setting.

Corollary 2. Let $U \in\left(\mathscr{S}^{\alpha}\right)^{\prime}\left(\mathbf{R}^{\prime}\right)$. Then $U=\left(U_{1}+U_{2}\right)$ where $U_{1} \in\left(\mathscr{S}^{\alpha}\right)^{\prime}$ and $U_{2} \in\left(\mathscr{S}^{x}\right)^{\prime}$; there exist functions $f_{1}(z)$ and $f_{2}(z)$ which are analytic in $\operatorname{Im}(z)>m>0$ and $\operatorname{Im}(z)<(-m)<0$, respectively, and which satisfy

$$
\begin{gathered}
\left|f_{1}(z)\right| \leqq K_{m}(1+|z|)^{N} \exp (2 \pi \sigma|y|), \quad y=\operatorname{Im}(z)>m>0, \\
\left|f_{2}(z)\right| \leqq K_{m}(1+|z|)^{N} \exp (2 \pi \sigma|y|), \quad y=\operatorname{Im}(z)<(-m)<0,
\end{gathered}
$$

for all $\sigma>0 ;$ and

$$
\begin{aligned}
& f_{1}(z) \rightarrow U_{1} \\
& f_{2}(z) \rightarrow U_{2}
\end{aligned}
$$

in the weak $\left(\mathscr{S}^{\alpha}\right)^{\prime}$ topology as $y=\operatorname{Im}(z) \rightarrow 0 \pm$, respectively.

As we have noted in the paragraph preceding Theorem 6 , the cone $C=\left(C_{1} \cup C_{2}\right)$, where $C_{1}=(0, \infty)$ and $C_{2}=(-\infty, 0)$, satisfies the properties indicated in the hypothesis of Theorem 6 . (Note that $C_{1}^{*}=[0, \infty)$ and $C_{2}^{*}=(-\infty, 0]$.) The conclusions of Corollary 2 thus follow immediately from Theorem 6 , and we have that $U_{1}$ and $U_{2}$ are the Fourier transforms of elements in $\mathscr{S}_{\alpha}^{\prime}$ having support in $[0, \infty)$ and $(-\infty, 0]$, respectively.

\section{REFERENCES}

1. S. Bochner and W. T. Martin, Several Complex Variables, Princeton University Press, Princeton, N. J., 1948.

2. Richard D. Carmichael, Distributional boundary values of functions analytic in tubular radial domains, Indiana Univ. Math. J., 20 (1971), 843-853.

3. - Generalized Cauchy and Poisson integrals and distributional boundary values, SIAM J. Math. Anal., 4 (1973), 198-219.

4. F. Constantinescu, Analytic properties of nonstrictly localizable fields, J. Mathematical Phys., 12 (1971), 293-298.

5. F. Constantinescu and W. Thalheimer, Euclidean Green's functions for Jaffe fields, preprint, 1974.

6. R. E. Edwards, Functional Analysis, Holt, Rinehart, and Winston, New York, 1965.

7. A. Friedman, Generalized Functions and Partial Differential Equations, Prentice-Hall, Englewood Cliffs, N. J., 1963.

8. I. M. Gel'fand and G. E. Shilov, Generalized functions, vol.1, Academic Press, New York, 1964.

9. - Generalized functions, vol. 2, Academic Press, New York, 1968.

10. _ Generalized functions, vol. 3, Academic Press, New York, 1967.

11. A. Rieckers, University of Munich preprint, 1968.

12. L. Schwartz, Théorie des distributions, Hermann, Paris, 1966. 
13. Mathematics for the Physical Sciences, Addison-Wesley, Reading, Mass., 1966.

14. V. S. Vladimirov, Methods of the theory of functions of several complex variables, M.I.T. Press, Cambridge, Mass., 1966.

Received March 26, 1974. The first author wishes to thank the Department of Mathematics, University of California, Davis, for its financial support in the summer of 1973, during which time the work on this paper began.

WAKE FOREST UNIVERSITY

AND

UNIVERSITY OF CALIFORNIA, DAVIS 


\section{PACIFIC JOURNAL OF MATHEMATICS \\ EDITORS}

\author{
Richard ARENS (Managing Editor) \\ University of California \\ Los Angeles, California 90024
}

\author{
R. A. Beaumont \\ University of Washington \\ Seattle, Washington 98105
}

\section{J. DugundJI}

Department of Mathematics

University of Southern California

Los Angeles, California 90007

D. Gilbarg and J. Milgram

Stanford University

Stanford, California 94305

\section{ASSOCIATE EDITORS}
E. F. BECKENBACH
B. H. NeumanN
F. WOLF
K. YoshidA

\section{SUPPORTING INSTITUTIONS}

\author{
UNIVERSITY OF BRITISH COLUMBIA \\ CALIFORNIA INSTITUTE OF TECHNOLOGY \\ UNIVERSITY OF CALIFORNIA \\ MONTANA STATE UNIVERSITY \\ UNIVERSITY OF NEVADA \\ NEW MEXICO STATE UNIVERSITY \\ OREGON STATE UNIVERSITY \\ UNIVERSITY OF OREGON \\ OSAKA UNIVERSITY
}

\author{
UNIVERSITY OF SOUTHERN CALIFORNIA \\ STANFORD UNIVERSITY \\ UNIVERSITY OF TOKYO \\ UNIVERSITY OF UTAH \\ WASHINGTON STATE UNIVERSITY \\ UNIVERSITY OF WASHINGTON \\ AMERICAN MATHEMATICAL SOCIETY
}

The Supporting Institutions listed above contribute to the cost of publication of this Journal, but they are not owners or publishers and have no responsibility for its contents or policies.

Mathematical papers intended for publication in the Pacific Journal of Mathematics should be in typed form or offset-reproduced (not dittoed), double spaced with large margins. Underline Greek letters in red, German in green, and script in blue. The first paragraph or two must be capable of being used separately as a synopsis of the entire paper. Items of the bibliography should not be cited there unless absolutely necessary, in which case they must be identified by author and Journal, rather than by item number. Manuscripts, in duplicate, may be sent to any one of the four editors. Please classify according to the scheme of Math. Reviews, Index to Vol. 39. All other communications should be addressed to the managing editor, or Elaine Barth, University of California, Los Angeles, California, 90024.

100 reprints are provided free for each article, only if page charges have been substantially paid. Additional copies may be obtained at cost in multiples of 50 .

The Pacific Journal of Mathematics is issued monthly as of January 1966. Regular subscription rate: $\$ 72.00$ a year (6 Vols., 12 issues). Special rate: $\$ 36.00$ a year to individual members of supporting institutions.

Subscriptions, orders for back numbers, and changes of address should be sent to Pacific Journal of Mathematics, 103 Highland Boulevard, Berkeley, California, 94708.

PUBLISHED BY PACIFIC JOURNAL OF MATHEMATICS, A NON-PROFIT CORPORATION Printed at Jerusalem Academic Press, POB 2390, Jerusalem, Israel.

$$
\begin{gathered}
\text { Copyright (c) } 1975 \text { Pacific Journal of Mathematics } \\
\text { All Rights Reserved }
\end{gathered}
$$




\section{Pacific Journal of Mathematics}

Vol. 56, No. 2 December, 1975

Ralph Alexander, Generalized sums of distances .................. 297

Zvi Arad and George Isaac Glauberman, A characteristic subgroup of a group of odd order ............................... 305

B. Aupetit, Continuité du spectre dans les algèbres de Banach avec involution ........................................ 321

Roger W. Barnard and John Lawson Lewis, Coefficient bounds for some classes of starlike functions . ...........................

Roger W. Barnard and John Lawson Lewis, Subordination theorems for some classes of starlike fumctions . .................... 333

Ladislav Bican, Preradicals and injectivity .................. 367

James Donnell Buckholtz and Ken Shaw, Series expansions of analytic

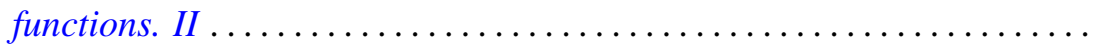

Richard D. Carmichael and E. O. Milton, Distributional boundary values in

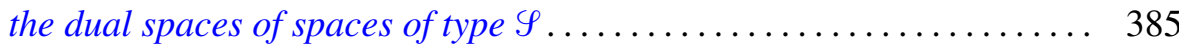

Edwin Duda, Weak-unicoherence ............................ 423

Albert Edrei, The Padé table of functions having a finite number of essential singularities ........................................

Joel N. Franklin and Solomon Wolf Golomb, A function-theoretic approach to the study of nonlinear recurring sequences ................ 455

George Isaac Glauberman, On Burnside's other $p^{a} q^{b}$ theorem 469

Arthur D. Grainger, Invariant subspaces of compact operators on topological vector spaces .........................

Jon Craig Helton, Mutual existence of sum and product integrals .

Franklin Takashi Iha, On boundary functionals and operators with finite-dimensional null spaces

Gerald J. Janusz, Generators for the Schur group of local and global number fields

A. Katsaras and Dar-Biau Liu, Integral representations of weakly compact operators.

W. J. Kim, On the first and the second conjugate points .

Charles Philip Lanski, Regularity and quotients in rings with involution ....

Ewing L. Lusk, An obstruction to extending isotopies of piecewise linear manifolds.

Saburou Saitoh, On some completenesses of the Bergman kernel and the Rudin kernel..

Stephen Jeffrey Willson, The converse to the Smith theorem for $Z_{p}$-homology spheres. 